\title{
Quantum physics exploring gravity in the outer solar system: the SAGAS project
}

\author{
P. Wolf • Ch. J. Bordé • A. Clairon • L. Duchayne • A. Landragin • \\ P. Lemonde - G. Santarelli · W. Ertmer - E. Rasel • F. S. Cataliotti · \\ M. Inguscio • G. M. Tino • P. Gill • H. Klein • S. Reynaud • C. Salomon • \\ E. Peik • O. Bertolami - P. Gil · J. Páramos • C. Jentsch • U. Johann • \\ A. Rathke · P. Bouyer • L. Cacciapuoti - D. Izzo $\cdot$ P. De Natale · \\ B. Christophe · P. Touboul - S. G. Turyshev • J. Anderson • M. E. Tobar • \\ F. Schmidt-Kaler · J. Vigué - A. A. Madej - L. Marmet • M.-C. Angonin • \\ P. Delva · P. Tourrenc • G. Metris · H. Müller • R. Walsworth • Z. H. Lu • \\ L. J. Wang • K. Bongs • A. Toncelli • M. Tonelli • H. Dittus • C. Lämmerzahl • \\ G. Galzerano · P. Laporta $\cdot$ J. Laskar · A. Fienga · F. Roques $\cdot$ K. Sengstock
}

Received: 30 November 2007 / Accepted: 17 July 2008 / Published online: 4 September 2008

(C) Springer Science + Business Media B.V. 2008

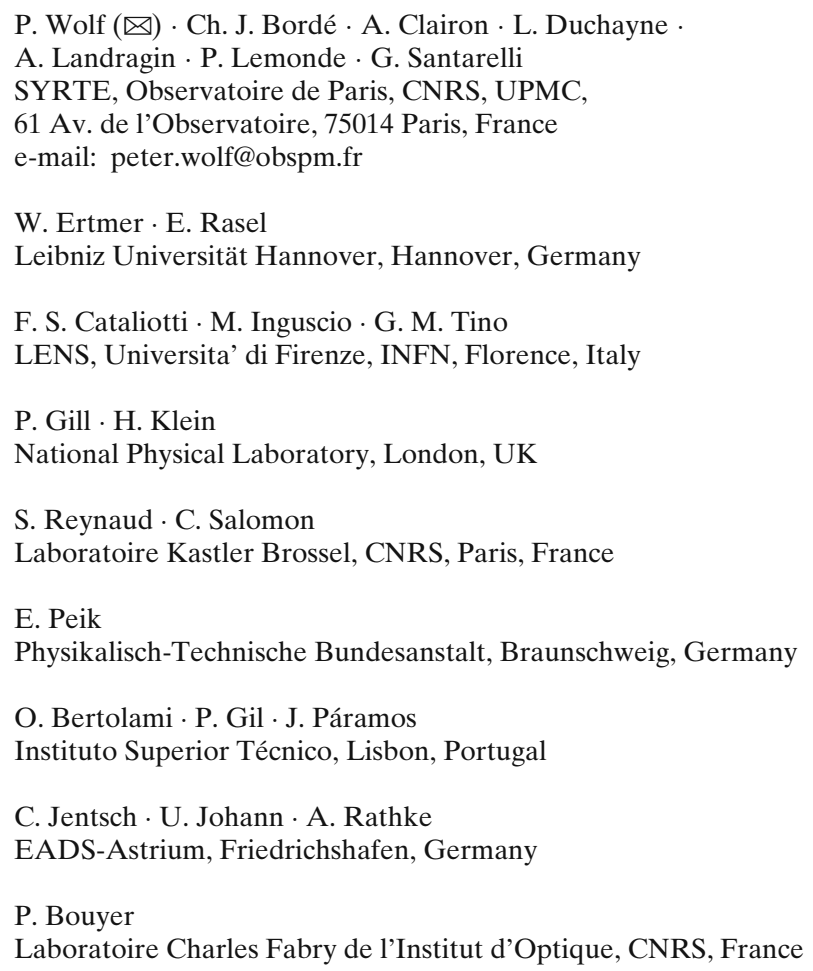


Abstract We summarise the scientific and technological aspects of the Search for Anomalous Gravitation using Atomic Sensors (SAGAS) project, submitted to ESA in June 2007 in response to the Cosmic Vision 2015-2025 call for proposals. The proposed mission aims at flying highly sensitive atomic sensors (optical clock, cold atom accelerometer, optical link) on a Solar System escape trajectory in the 2020 to 2030 time-frame. SAGAS has numerous science objectives in fundamental physics and Solar System science, for example numerous tests of general relativity and the exploration of the Kuiper belt. The combination of highly sensitive atomic sensors and of the laser link well adapted for large distances will allow measurements with unprecedented accuracy and on scales never reached before. We present the proposed mission in some detail, with particular emphasis on the science goals and associated measurements and technologies.

Keywords Fundamental physics • Trans Neptunian objects • Optical link • Atomic clock • Atomic accelerometer

L. Cacciapuoti · D. Izzo

ESTEC, ESA, Noordwijk, The Netherlands

P. De Natale

INOA-CNR, Firenze, Italy

B. Christophe $\cdot$ P. Touboul

ONERA, Chatillon, France

S. G. Turyshev

Jet Propulsion Laboratory, NASA, Pasadena, CA, USA

J. Anderson

Jet Propulsion Laboratory, California Institute of Technology, Pasadena, CA, USA

M. E. Tobar

University of Western Australia, Perth, Australia

F. Schmidt-Kaler

Universität Ulm, Ulm, Germany

J. Vigué

LCAR, Université Toulouse III, CNRS, Toulouse, France

A. A. Madej

National Research Council of Canada (NRC-CNRC), Ottawa, Canada

L. Marmet

Institut des Étalons Nationaux de Mesures, CNRC, Ottawa, Canada

M.-C. Angonin $\cdot$ P. Delva $\cdot$ P. Tourrenc

ERGA/LERMA, Université Paris 6, Paris, France

G. Metris

GEMINI, Observatoire de la Côte Azur, CNRS, Nice, France 


\section{Abbreviations}

$\begin{array}{ll}\text { S/C } & \text { spacecraft } \\ \text { PPN } & \text { parameterized post Newtonian } \\ \text { PSD } & \text { power spectral density } \\ \text { CMB } & \text { cosmic microwave background } \\ \text { DSN } & \text { deep space network } \\ \text { USO } & \text { ultra stable oscillator } \\ \text { TRL } & \text { technology readiness level } \\ & \text { (http://sci.esa.int/science-e/www/object/index. } \\ & \text { cfm?fobjectid=37710) } \\ \text { SNR } & \text { signal to noise ratio } \\ \text { SLR } & \text { satellite laser ranging } \\ \text { LLR } & \text { lunar laser ranging } \\ \text { CCD } & \text { charge coupled device } \\ \text { FoV } & \text { field of view } \\ \text { RTG } & \text { radioisotope thermoelectric generator } \\ \text { ECDL } & \text { extended cavity diode laser }\end{array}$

H. Müller

Stanford University, Stanford, CA, USA

R. Walsworth

Harvard University, Cambridge, MA, USA

Z. H. Lu $\cdot$ L. J. Wang

University of Erlangen, Erlangen, Germany

K. Bongs

University of Birmingham, Birmingham, UK

A. Toncelli $\cdot$ M. Tonelli

NEST, INFM-CNR, Universita' di Pisa, Pisa, Italy

H. Dittus · C. Lämmerzahl

ZARM, University of Bremen, Bremen, Germany

G. Galzerano · P. Laporta

Politecnico di Milano, Milan, Italy

J. Laskar

IMCCE, Observatoire de Paris, CNRS, Paris, France

A. Fienga

Observatoire de Besançon, Besançon, France

F. Roques

LESIA, Observatoire de Paris, CNRS, Paris, France

K. Sengstock

Universität Hamburg, Hamburg, Germany 


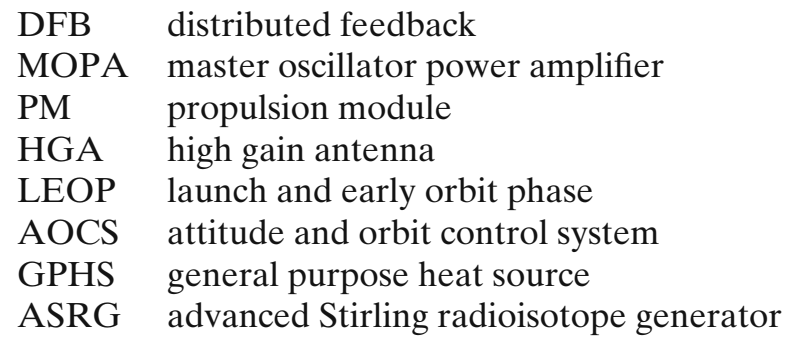

\section{Introduction}

The SAGAS mission will study all aspects of large scale gravitational phenomena in the Solar System using quantum technology, with science objectives in fundamental physics and Solar System exploration. The large spectrum of science objectives makes SAGAS a unique combination of exploration and science, with a strong basis in both programs. The involved large distances (up to $53 \mathrm{AU}$ ) and corresponding large variations of gravitational potential combined with the high sensitivity of SAGAS instruments serve both purposes equally well. For this reason, SAGAS brings together traditionally distant scientific communities ranging from atomic physics through experimental gravitation to planetology and Solar System science.

The payload will include an optical atomic clock optimised for long term performance, an absolute accelerometer based on atom interferometry and a laser link for ranging, frequency comparison and communication. The complementary instruments will allow highly sensitive measurements of all aspects of gravitation via the different effects of gravity on clocks, light, and the free fall of test bodies, thus effectively providing a detailed gravitational map of the outer Solar System whilst testing all aspects of gravitation theory to unprecedented levels.

The SAGAS accelerometer is based on cold Cs atom technology derived to a large extent from the PHARAO space clock built for the Atomic Clock Ensemble in Space (ACES) mission [1]. The PHARAO engineering model has recently been tested with success, demonstrating the expected performance and robustness of the technology. The accelerometer will only require parts of PHARAO (cooling and trapping region) thereby significantly reducing mass and power requirements. The expected sensitivity of the accelerometer is 1.3 $\times 10^{-9} \mathrm{~m} / \mathrm{s}^{2} \mathrm{~Hz}^{-1 / 2}$ with an absolute accuracy (bias determination) of $5 \times$ $10^{-12} \mathrm{~m} / \mathrm{s}^{2}$.

The SAGAS clock will be an optical clock based on trapped and laser cooled single ion technology as pioneered in numerous laboratories around the world. In the present proposal it will be based on a $\mathrm{Sr}^{+}$ion with a clock wavelength of $674 \mathrm{~nm}$. The expected stability of the SAGAS clock is $1 \times 10^{-14} / \sqrt{ } \tau$ (with $\tau$ the integration time), with an accuracy in realising the unperturbed ion frequency of $1 \times 10^{-17}$. The best optical single ion ground clocks presently show stabilities below $3 \times 10^{-15} / \sqrt{ } \tau$, slightly better than the one assumed for the SAGAS 
clock, and only slightly worse accuracies $\left(2 \times 10^{-17}\right)$. So the technology challenges facing SAGAS are not so much the required performance, but the development of reliable and space qualified systems, with reduced mass and power consumption.

The optical link is using a high power $(1 \mathrm{~W})$ laser locked to the narrow and stable frequency provided by the optical clock, with coherent heterodyne detection on the ground and on board the spacecraft (S/C). It serves the multiple purposes of comparing the SAGAS clock to ground clocks, providing highly sensitive Doppler measurements for navigation and science, and allowing data transmission together with timing and coarse ranging. It is based on a $40 \mathrm{~cm}$ space telescope and $1.5 \mathrm{~m}$ ground telescopes (similar to lunar laser ranging stations). The main challenges of the link will be the required pointing accuracy ( 0.3 ") and the availability of space qualified, robust $1 \mathrm{~W}$ laser sources at $674 \mathrm{~nm}$. Quite generally, laser availability and reliability will be the key to achieving the required technological performances, for the clock as well as the optical link (see "Section 6.2").

For this reason a number of different options have been considered for the clock/link laser wavelength, with several other ions that could be equally good candidates (e.g. $\mathrm{Yb}^{+}$at $435 \mathrm{~nm}$ and $\mathrm{Ca}^{+}$at $729 \mathrm{~nm}$ ). Given present laser technology, $\mathrm{Sr}^{+}$was preferred, but this choice could be revised depending on laser developments over the next years. We also acknowledge the possibility that femtosecond laser combs might be developed for space applications in the near future, which would open up the option of using either ion with existing space qualified 1,064 nm Nd:YAG lasers for the link.

More generally, SAGAS technology takes advantage of the important heritage from cold atom technology used in PHARAO and laser link technology designed for Lasers Interferometric Space Antenna (LISA). It will provide an excellent opportunity to develop those technologies for general use, including development of the ground segment (Deep Space Network telescopes and optical clocks), that will allow such technologies to be used in many other mission configurations for precise timing, navigation and broadband data transfer throughout the Solar System.

In summary, SAGAS offers a unique opportunity for a high profile deep space mission with a large spectrum of science objectives in Solar System exploration and fundamental physics, and the potential for a major breakthrough in our present conception of physics, the Solar System and the universe as a whole.

\section{Science objectives}

SAGAS will carry out a large number of tests of fundamental physics, and gravitation in particular, at scales only attainable in a deep space experiment. The unique combination of onboard instruments will allow two to five orders of magnitude improvement on many tests of special and general relativity, as well as a detailed exploration of a possible anomalous scale dependence 
of gravitation. It will also provide detailed information on the Kuiper belt mass distribution and determine the mass of Kuiper belt objects and possibly discover new ones. During the transits, the mass and mass distribution of the Jupiter system will be measured with unprecedented accuracy. The science objectives are summarised in Table 1 below. They are investigated in more detail in the following subsections based on estimated measurement uncertainties of the different observables.

\subsection{Measurements and observables}

SAGAS will provide three fundamental measurements: the accelerometer readout and the two frequency differences (measured on ground and on board the satellite) between the incoming laser signal and the local optical clock. Auxiliary measurements are the timing of emitted/received signals on board and on the ground, which are used for ranging and time tagging of data. The high precision science observables will be deduced from the fundamental measurements by combining the measurements to obtain information on either the frequency difference between the clocks or the Doppler shift of the transmitted signals. The latter gives access to the relative satellite-ground velocity, from which the gravitational trajectory of the satellite can be deduced by correcting non-gravitational accelerations $\left(\mathbf{a}_{\mathrm{NG}}\right)$ using the accelerometer readings. Then the three science observables are

$\begin{array}{ll}\text { Relative frequency : } & y \equiv \partial_{t} \tau_{S}-\partial_{t} \tau_{G} \\ \text { Doppler shift : } & D_{v} \equiv\left(v_{r}-v_{e}\right) / v_{0} \\ \text { Non-gravitational acceleration : } & \mathbf{a}_{\mathrm{NG}}\end{array}$

where $\tau_{i}$ is proper time at the position of the space and ground clock respectively, $t$ is coordinate time, $v_{i}$ is the received, emitted, and nominal proper frequency of a photon, and $D_{v}$ is corrected for non-gravitational satellite motion.

In the following, we assume that Earth station motion and its local gravitational potential can be known and corrected to uncertainty levels below $10^{-17}$ in relative frequency $(<10 \mathrm{~cm}$ on geocentric distance), which, although challenging, are within present capabilities. For the Solar System parameters this requires $10^{-9}$ relative uncertainty for the ground clock parameters (GM and $r$ of Earth), also achieved at present [2], and less stringent requirements for the satellite.

For long term integration and the determination of an acceleration bias, the limiting factor will then be the accelerometer noise and absolute uncertainty (bias determination), shown in Table 2. More generally, modelling of nongravitational accelerations will certainly allow some improvement on the long term limits imposed by the accelerometer noise and absolute uncertainty, but is not taken into account in Table 2. Also, depending on the science goal and corresponding signal, the ground and on-board data can be combined in a way to optimise the $\mathrm{S} / \mathrm{N}$ ratio (see [3] for details). For example, in the Doppler observable $D_{v}$ the contribution of one of the clocks (ground or space) can be 


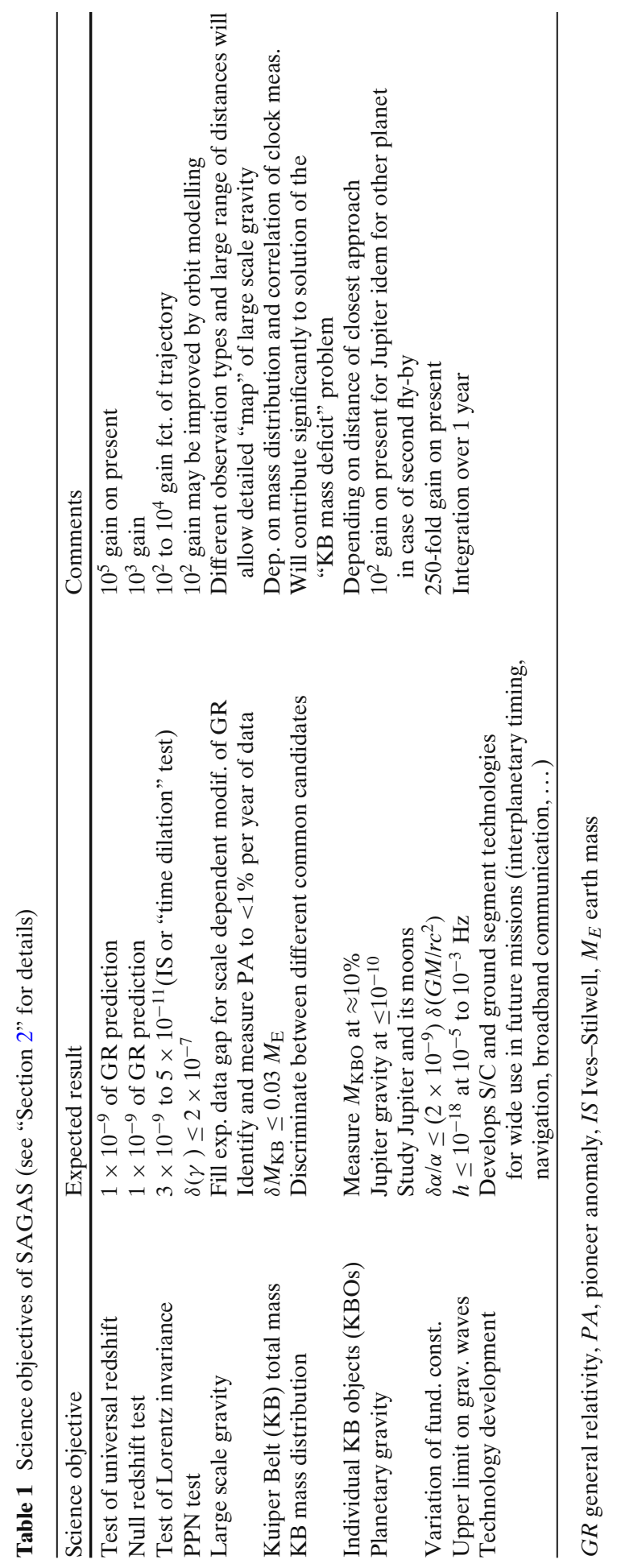


Table 2 SAGAS uncertainties on science observables

\begin{tabular}{|c|c|c|c|}
\hline & Noise PSD $/ \mathrm{Hz}^{-1}$ & Bias & Comments \\
\hline$y$ & $\left(2 \times 10^{-28}+9 \times 10^{-24} f^{2}\right)$ & $10^{-17}$ & \\
\hline$D_{v}$ & $\left(4.5 \times 10^{-37} f^{-2}+1 \times 10^{-28}+9 \times 10^{-24} f^{2}\right)$ & $\left(10^{-17}\right)$ & $\begin{array}{l}\text { Bias determination } \\
\text { limited by accelerometer } \\
\text { and orbit modelling, } \\
10^{-17} \text { is clock limit }\end{array}$ \\
\hline $\mathbf{a}_{\mathrm{NG}}$ & $1.6 \times 10^{-18}\left(\mathrm{~m} / \mathrm{s}^{2}\right)^{2}$ & $5 \times 10^{-12} \mathrm{~m} / \mathrm{s}^{2}$ & \\
\hline
\end{tabular}

Note that stated Power Spectral Densities are valid for integration down to the bias uncertainties

made negligible by combining signals such that one has coincidence of the "up" and "down" signals on board or on the ground.

We will use a mission profile with a nominal mission lifetime of 15 years and the possibility of an extended mission to 20 years if instrument performance and operation allow this. In that time frame, the trajectory allows the satellite to reach a heliocentric distance of $39 \mathrm{AU}$ in nominal mission and $53 \mathrm{AU}$ with extended duration.

\subsection{Test of the gravitational redshift and of Lorentz invariance}

In General Relativity (GR), the frequency difference of two ideal clocks is (to first order in the weak field approximation)

$$
\frac{d \tau_{S}}{d t}-\frac{d \tau_{G}}{d t} \approx \frac{w_{G}-w_{S}}{c^{2}}+\frac{v_{G}^{2}-v_{S}^{2}}{2 c^{2}}+O\left(c^{-4}\right)
$$

with $w$ the Newtonian gravitational potential at the location of the clocks and $v$ their coordinate velocity. In theories different from GR the relation Eq. 2 is modified, leading to different time and space dependence of the frequency difference. This can be tested by comparing two clocks at distant locations (different values of $w$ and $v$ ) via exchange of an electromagnetic signal. The SAGAS trajectory (large potential difference) and low uncertainty on the observable (directly the difference in Eq. 2) allows a relative uncertainty on the redshift determination given by the $10^{-17}$ clock bias divided by the maximum value of $\left(w_{G}-w_{S}\right) / c^{2}$. For a distance of $50 \mathrm{AU}$ this corresponds to a test with a relative uncertainty of $1.0 \times 10^{-9}$, an improvement by about five orders of magnitude on the uncertainty obtained by the most sensitive experiment at present [4].

Additionally, the mission also provides the possibility of testing the velocity term in Eq. 2, which amounts to a test of Special Relativity (Ives-Stilwell test), and thus of Lorentz invariance. Towards the end of the nominal mission, this term is about $4 \times 10^{-9}$ and can therefore be measured by SAGAS with $3 \times$ $10^{-9}$ relative uncertainty. The best present limit on this type of test is $2.2 \times$ $10^{-7}[5]$, so SAGAS will allow an improvement by a factor $\approx 70$. Considering a particular preferred frame, usually taken as the frame in which the $3 \mathrm{~K}$ cosmic background radiation is isotropic, one can set an even more stringent 
limit. In that case a putative effect will be proportional to $\left(\mathbf{v}_{\mathrm{S}}-\mathbf{v}_{\mathrm{G}}\right) \cdot \mathbf{v}_{\mathrm{Sun}} /$ $c^{2}$ (cf. [5]), where $\mathbf{v}_{\text {Sun }}$ is the velocity of the Sun through the CMB frame $(\approx 350 \mathrm{~km} / \mathrm{s})$. Then SAGAS will allow a measurement with about $5 \times 10^{-11}$ relative uncertainty, which corresponds to more than three orders of magnitude improvement on the present limit. Note that Ives-Stilwell experiments also provide the best present limit on a particularly elusive parameter $\left(\kappa_{\mathrm{tr}}\right)$ of the Lorentz violating Standard Model Extension (SME) photon sector [6], hence SAGAS also allows for the same factor 70 to $10^{3}$ improvement on that parameter.

\subsection{Tests of parameterised post-Newtonian gravity}

The PPN formalism describing a large class of metric theories of gravitation including GR in the weak field regime is well known (see e.g. [7]) and has been extensively tested in the Solar System. The two most common parameters of the PPN framework are the Eddington parameters $\beta$ and $\gamma$, both equal to 1 in GR. Present limits on $\gamma$ are obtained from measurements on light propagation such as light deflection and Shapiro delay. The tightest limit was deduced from Doppler ranging to the Cassini mission during solar occultation in June 2002 yielding $\gamma=1+(2.1 \pm 2.3) \times 10^{-5}$ [8]. SAGAS will carry out similar measurements during solar conjunctions, however with improved sensitivity and at optical rather than radio frequencies, which significantly minimizes errors due to the solar corona and the Earth's ionosphere. When the laser of the SAGAS link passes close to the sun the gravitational Shapiro delay leads to a maximum modification of the Doppler observable $\delta D_{v}$ of about $8.4 \times 10^{-10}$. When combining the on board and ground measurements such that the "up" and "down" signals coincide at the satellite (classical Doppler ranging type measurement) the noise from the on-board clock cancels to a large extent and one is left with noise from the accelerometer, the ground clock, and the atmosphere. We assume that for a 20 day measurement around occultation the accelerometer is operated in $1 \mathrm{D}$ along the direction of signal propagation (of interest here) leading to a factor of $\sqrt{ } 3$ improvement on its sensitivity given in "Section 3.1.2" and Table 2. We assume that ground optical clocks improve by about a factor 6 in stability with respect to best present performances to $\sigma_{\mathrm{y}}(\tau) \approx 5 \times 10^{-16} / \sqrt{ } \tau$ in terms of Allan variance (very likely by the time SAGAS is launched). Finally we assume atmospheric noise from turbulence and variations in temperature, pressure and humidity as described in "Section 3.3.4." Optimal filtering of the Shapiro delay signal in the total noise during a 10 day measurement starting just after occultation leads to an uncertainty $\delta(\gamma) \leq 1.1 \times 10^{-7}$. Considering also the 10 day measurement just before occultation allows another $\sqrt{ } 2$ gain, and $N$ occultations during the 20 year duration allow another gain of $\sqrt{ } N$. However, observations are likely to be incomplete over the 20 days around occultation (data gaps due to loss of lock e.g. from atmospheric fluctuations). Allowing for a factor $\approx 2$ loss (about a factor 4 loss in observation time) we thus conservatively estimate our overall 
uncertainty on the determination of $\gamma$ from a single occultation at $2 \times 10^{-7}$, with some potential for improvement with several occultations.

\subsection{Exploring large scale gravity}

Experimental tests of gravity show a good agreement with GR at scales ranging from a millimeter in laboratory experiments to the size of planetary orbits. Meanwhile, most theoretical models aimed at inserting GR within the quantum framework predict observable modifications at smaller and/or larger scales. Anomalies observed in the rotation curves of galaxies or in the relation between redshifts and luminosities of supernovae are ascribed to "dark matter" and "dark energy" components, the nature of which remains unknown. These dark components, which could constitute $96 \%$ of the content of the Universe, have not been detected by non-gravitational means to date. As the observed anomalies could also be consequences of modifications of GR at galactic or cosmological scales, it is extremely important to test the laws of gravity at the largest possible distances.

Tracking the orbit of the Pioneer 10/11 probes during their extended missions allowed for the largest scaled experimental test of gravity ever performed. The results failed to reproduce the expected variation of the gravity force with distance [9-13]. Precisely, the analysis of the radio-metric tracking data from the probes at distances between 20-70 AU from the Sun has shown the presence of an anomalous, nearly constant drift of the Doppler shift, interpreted as an unexpected acceleration of the order of $1 \mathrm{~nm} / \mathrm{s}^{2}$, towards the Sun. The observation of this "Pioneer anomaly" (PA) has stimulated significant efforts to find explanations in terms of systematic effects on board the spacecraft or in its environment. The inability to explain the anomalous behavior of the Pioneer spacecraft with conventional physics [9-13] has contributed to the growing discussion about its origin, a discussion which is still ongoing [14]. It has also motivated an interest in flying new probes to the distances where the anomaly was first discovered, that is beyond the Saturn orbit, and studying gravity with modern techniques.

Over the past years a large number of theoretical frameworks that allow for a scale (distance) dependent modification of GR have been suggested, e.g. generalized metric extensions of GR, Modified Newtonian Dynamics, TensorVector-Scalar-theory, Metric-Skew-Tensor Gravity, $f(R)$ modified gravity theories, String theory and Cosmology motivated frameworks, Braneworld scenarios, and many others. It is far beyond the scope of this paper to study the effects of those frameworks on the SAGAS observables. A study under a number of conventional and "new physics" hypotheses allows two main conclusions:

- With 1 year of integration all SAGAS observables allow a measurement of any effect of the size of the PA with a relative uncertainty of better than $1 \%$. This will allow a "mapping" of any anomalous scale dependence over the mission duration and corresponding distances. 
- The complementary observables available on SAGAS allow a good discrimination between different hypotheses thereby not only measuring a putative effect but also allowing an identification of its origin.

In summary, SAGAS offers the possibility to constrain a significant number of theoretical approaches to scale dependent modifications of GR. Given the complementary observables available on SAGAS the obtained measurements will provide a rich testing ground for such theories with the potential for major discoveries that may well lead to major results on relativity and physics as a whole.

\subsection{Exploring outer solar system masses}

The Kuiper belt (KB) is a collection of masses, remnant of the circumsolar disk where giant planets of the solar system formed 4.6 billion years ago. Precise measurements of its mass distribution would significantly improve our understanding of planet formation not only in the Solar System but also in recently discovered planetary systems. The exceptional sensitivity and versatility of SAGAS for measuring gravity can be used to study the sources of gravitational fields in the outer solar system, and in particular the class of Trans Neptunian Objects (TNOs), of which those situated in the Kuiper belt have been the subject of intense interest and study over the last years [15]. Observation of Kuiper belt objects (KBOs) from the Earth is difficult due to their relatively small size and large distance, and estimates of their masses and distribution are accordingly inaccurate. Estimates of the total KB mass from the discovered objects $(\approx 1,000 \mathrm{KBOs})$ range from 0.01 to 0.1 Earth masses, whereas in-situ formation of the observed KBOs would require three orders of magnitude more solid material in a dynamically cold disk.

A dedicated probe like SAGAS will help discriminating different models of the spatial distribution of the Kuiper Belt and determining its total mass. Figure 1 shows the relative frequency shift between the ground and space clock due to the Kuiper Belt gravitational potential $\delta y=w_{\mathrm{KB}}(r) / c^{2}$, as a function of heliocentric distance of the satellite, and for several models of the spatial distribution of $M_{\mathrm{KP}}$ (see [16, 17] for details).

Apart from the weaker detectability of the non-uniform disk mass distribution, the remaining models can be essentially detected from distances beyond $15 \mathrm{AU}$, and well discriminated at around $40 \mathrm{AU}$, well within the mission target distance. Furthermore, a complete "scan" over all distances available during the mission not only allows the determination of the shape of the curves shown on Fig. 1 and hence the mass distribution, but also the amplitude i.e. the total mass $M_{\mathrm{KP}}$. The accuracy of that will obviously depend on the distribution. For example, in the "two rings" distribution SAGAS will determine $M_{\mathrm{KP}}$ with an uncertainty of at least $10 \%$ i.e. about 0.03 Earth masses. In a more detailed analysis one would fit all measurements during the mission to the candidate curve thereby likely decreasing the overall uncertainty by at most (depending on correlations of individual measurements) $\sqrt{ } \mathrm{N}$ where $\mathrm{N}$ is the number of 


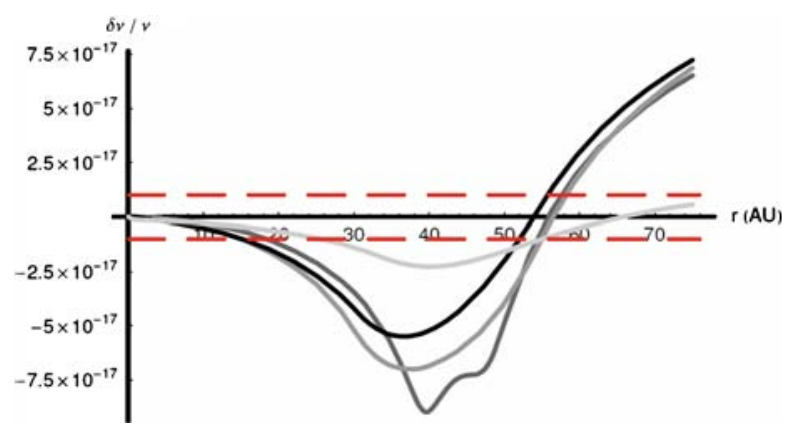

Fig. 1 Relative frequency shift as a function of heliocentric distance from different Kuiper belt mass distributions, with a total mass of $M_{\mathrm{KP}}=0.3 M_{\mathrm{E}}$ [16]: Torus (black), Two rings (dark grey), Uniform disk (medium grey), Non-uniform disk (light grey). The dashed red lines indicate the uncertainties of the SAGAS clock

measurements. Given that the clock noise integrates to $10^{-17}$ in about 10 days and for the $\approx 14$ years travel from $15 \mathrm{AU}$ to the end of the extended mission (53 AU), this is another potential factor $\approx 20$ improvement.

The SAGAS frequency observable $y$ is well suited to study the large, diff use, statistical mass distribution of KBOs essentially due to its sensitivity directly to the gravitational potential $(1 / r$ dependence $)$, rather than the acceleration $\left(1 / r^{2}\right.$ dependence). The large diff use signal masks any signal from individual KBOs. When closely approaching one of the objects, the crossover between acceleration sensitivity (given by the $5 \times 10^{-12} \mathrm{~m} / \mathrm{s}^{2}$ uncertainty on $\mathbf{a}_{\mathrm{NG}}$ ) and the frequency sensitivity $\left(10^{-17}\right.$ uncertainty on $\left.\mathrm{GM} /\left(r c^{2}\right)\right)$ for an individual object is situated at about 1.2 AU. Below that distance the acceleration measurement is more sensitive than the frequency measurement. This suggests a procedure to study individual objects using the SAGAS observables: use the satellite trajectory (corrected for $\mathbf{a}_{\mathrm{NG}}$ ) to study the gravity from a close object and subtract the diffuse background from all other KBOs using the frequency measurement. Investigating several known KBOs within the reach of SAGAS [18] shows that their masses can be determined at the percent level when approaching any of them to $0.2 \mathrm{AU}$ or less. Of course, this also opens the way towards the discovery of new such objects, too small to be visible from the Earth.

Similarly, during a planetary flyby the trajectory determination (corrected for $\mathbf{a}_{\mathrm{NG}}$ ) will allow the determination of the gravitational potential of the planetary system. The planned Jupiter flyby with a closest approach of $\approx 600,000 \mathrm{~km}$ will improve present knowledge of Jovian gravity by more than two orders of magnitude.

\subsection{Variation of fundamental constants}

Spatial and/or temporal variations of fundamental constants constitute another violation of Local Position Invariance and thus of GR. Over the past few years, there has been great interest in that possibility (see e.g. [19] for a review), 
spurred on the one hand by models for unification theories of the fundamental interactions where such variations appear quite naturally, and on the other hand by recent observational claims of a variation of different constants over cosmological timescales [20,21]. Such variations can be searched for with atomic clocks, as the involved transition frequencies depend on combinations of fundamental constants and in particular, for the optical transition of the SAGAS clock, on the fine structure constant $\alpha$. Such tests take two forms: searches for a drift in time of fundamental constants, or for a variation of fundamental constants with ambient gravitational field. The latter tests for a nonuniversal coupling between ambient gravity and non-gravitational interactions (clearly excluded by GR) and is well measured by SAGAS, because of the large change in gravitational potential during the mission.

For example, some well-known string theory based models associate a scalar field such as the dilation to the standard model [22-25]. Such scalar fields would couple to ordinary matter and thus their non-zero value would introduce a variation of fundamental constants, in particular $\alpha$ of interest here. The nonzero value of such scalar fields could be of cosmological origin, leading to a constant drift in time of fundamental constants, and/or of local origin, i.e. taking ordinary matter as its source [22]. In the latter case one would observe a variation of fundamental constants with the change in local gravitational potential, which can be parameterized in the simple form [22]

$$
\frac{\delta \alpha}{\alpha}=k_{\alpha} \delta\left(\frac{\mathrm{GM}}{r c^{2}}\right) .
$$

The difference in gravitational potential between the Earth and the SAGAS satellite at the end of nominal mission is about $\delta\left(\mathrm{GM} /\left(r c^{2}\right)\right) \approx 9.7 \times 10^{-9}$, which is 30 times more than the variation attainable on Earth. The $\mathrm{Sr}^{+}$optical transition used in the SAGAS clock has a sensitivity to the variation of $\alpha$ of $\approx 0.43$. When compared to a ground clock with $10^{-17}$ uncertainty, this yields a limit of $k_{\alpha}<2.4 \times 10^{-9}$, a factor 250 improvement over the best present limit [22].

\subsection{Upper limits on low frequency gravitational waves}

Doppler ranging to deep space missions provides the best upper limits available at present on gravitational waves $(\mathrm{GW})$ with frequencies of order $c / L$ where $L$ is the $\mathrm{S} / \mathrm{C}$ to ground distance i.e. in the $10^{-3}$ to $10^{-5} \mathrm{~Hz}$ range $[26,27]$, and even down to $<10^{-6} \mathrm{~Hz}$, albeit with lower sensitivity [27, 28]. The corresponding limits on GW are determined by the noise PSD of the Doppler ranging to the spacecraft for stochastic GW backgrounds [27, 28], filtered by the bandwidth of the observations when looking for GW with known signatures $[26,27]$. In the case of SAGAS, using optimal data combination allows a strain sensitivity of $\sim 10^{-14} / \sqrt{ } \mathrm{Hz}$ for stochastic sources in the range of $10^{-5}$ to $10^{-3} \mathrm{~Hz}$ [3]. When searching for $\mathrm{GW}$ with particular signatures in the $10^{-5}$ to $10^{-3} \mathrm{~Hz}$ frequency region, optimal filtering using a corresponding 
GW template will allow reaching strain sensitivities as low as $h \approx 10^{-18}$ with 1 year of data. This will improve on best present upper limits on GW in the $10^{-5}$ to $10^{-3} \mathrm{~Hz}$ frequency range by about four orders of magnitude. It is not expected at present that GW with sufficiently large amplitudes are found, still the results might serve as upper bounds for astrophysical and cosmological models of GW sources.

\subsection{Technology development}

SAGAS technology choices are based on cold atom and laser technology, both particularly adapted for tracking, timing and communication over large distances and measurement of DC (or very slowly varying) effects because of the absolute reference provided by the atoms. The mission takes advantage from important heritage on cold atom technology used in ACES/PHARAO and laser link technology designed for LISA. It will provide an excellent opportunity to develop those technologies for general use in interplanetary missions, including development of the ground segment (DSN telescopes and optical clocks) that will allow such technologies to be used in many other mission configurations for precise timing, navigation and broadband data transfer throughout the Solar System.

\section{Payload}

The SAGAS payload is composed of two instruments (accelerometer and optical clock) and the optical link which is used as a scientific instrument (frequency comparison and Doppler measurements), and also for data transfer. A particular feature of the SAGAS payload is the synergy between the different payload parts which significantly simplifies the overall design and reduces cost and technology development: The accelerometer also provides the absolute calibration of the quartz Ultra Stable Oscillator (USO) with respect to the Cs atoms, which in turn is used to generate all on-board RF signals used in the optical clock, the link and the time-tagging of observations. The optical clock and optical link share the same frequency, thereby avoiding use of a femtosecond frequency comb and allowing common use of some of the laser sources, with the clock providing a highly narrow and stable laser for the link. In short, the combination of on board instruments is not only optimal and complementary for the science objectives, but also close to ideal for the simplicity and coherence of the technology.

\subsection{Cold atom accelerometer}

To reach the scientific objectives of SAGAS, accurate measurements of accelerations along three orthogonal axes are required. The design payload and its characteristics arise from the development of the fields of cold atom 
physics and atom interferometry [29, 30]. Key technologies are identical to those already developed within the ACES project for the PHARAO payload. Compared to PHARAO, the SAGAS accelerometer shares identical key technologies and similar payload architecture and subsystems. Concerning issues related to atom interferometry, it also benefits from the different studies carried out within the HYPER project. Ground developments of cold atom interferometers have already shown performances comparable to state of the art optical interferometers [31, 32]. Measurement of the gravity acceleration is limited on Earth to a few parts in $10^{9}$ by environment effects: tides, atmospheric pressure and underground water fluctuations..., which vanish in a space environment. The intrinsic accuracy of cold atom interferometers makes them attractive for studies in fundamental physics, e.g. for the determination of the gravitational constant $G[33,34]$ or for the measurement of the Planck constant $\hbar[35,36]$, and for applications in the fields of geophysics and geodesy.

\subsubsection{Principle of operation and baseline choice}

The accelerometer is based on the use of cold atoms and Raman transitions for the manipulation of the atomic wave-packets. The atoms are alkaline atoms, which can be easily cooled using all solid-state semi-conductor diode lasers. The Raman transitions couple the two ground states of the alkaline atoms (noted $|\mathrm{g}\rangle$ and $|\mathrm{e}\rangle$ ) and can be realized by the same lasers as the cooling. The two Raman lasers are propagating in opposite directions and transfer a momentum $\hbar k$ to diffracted atoms (corresponding to a velocity of the order of $1 \mathrm{~cm} \mathrm{~s}^{-1}$ ). The phase shift due to acceleration is given by: $\Delta \phi=$ $-\mathbf{a} \cdot \mathbf{k} \cdot T^{2}[35,36]$. The sensitivity depends only on the wave vector $\mathbf{k}$ and the square of the time between pulses $T$. Each cycle (cooling, state preparation, interrogation sequence, detection) lasts $3 \mathrm{~s}$, for an interrogation time $T=1 \mathrm{~s}$. The three axes of acceleration are successively measured within the same vacuum tube using three orthogonal pairs of Raman lasers.

The two most advantageous choices of atomic species are $\mathrm{Rb}$ and $\mathrm{Cs}$, with a slight advantage for the latter stemming essentially from the better TRL coming from the PHARAO project. Thus Cs was chosen for the SAGAS accelerometer.

Different possible sources of cold atoms can be used for the atom interferometer: molasses, magneto-optical trap (MOT), ultra-cold sources from evaporative cooling in a magnetic or an optical trap (in degenerate state or not). The choice of an optical molasses has been driven by the simplicity, lower mass and power consumption whilst still allowing about $3 \times 10^{5}$ detected atoms, sufficient for the purposes of SAGAS.

\subsubsection{Acceleration measurement: noise and accuracy}

The sensitivity to acceleration per cycle is given by $1 /\left(k T^{2}\right.$.SNR), where SNR is the signal to noise ratio of the measurement. This sensitivity improves with 
the averaging time $\tau$ as $1 / \sqrt{ } \tau$. Different sources of noise limit the SNR: atom shot noise, phase noise between Raman lasers, acceleration noises. The latter is due essentially to $\mathrm{S} / \mathrm{C}$ vibrations stemming from unbalance of the AOCS fly wheels and was estimated using Bepi-Colombo mission studies.

It turns out that the limiting effect is the residual phase noise between Raman lasers due to the imperfections of the reference frequency at $9.2 \mathrm{GHz}$. By using the spectral noise density of the PHARAO flight model frequency reference (Quartz USO), we obtain a SNR $=200$ per shot (3 s total) leading to a sensitivity limit of $3 \times 10^{-10} \mathrm{~m} \mathrm{~s}^{-2}$ per shot. As the sensitivity improves with the square root of the measurement time, the required sensitivity $\left(5 \times 10^{-12} \mathrm{~m}\right.$ $\mathrm{s}^{-2}$ ) is obtained after only $3 \mathrm{~h}$ per axis, which gives $9 \mathrm{~h}$ for the three axes.

To achieve the requirement for SAGAS, the accelerometer has to be accurate at a level of $5 \times 10^{-12} \mathrm{~m} \mathrm{~s}^{-2}$, i.e. all acceleration biases need to be controlled at that level. Compared to ground experiments, the absence of gravity, and therefore of average velocity of the atomic cloud, allows reduction of most of the systematics. Moreover, some of the error sources decrease with increasing interaction time $T$, as they depend on the interaction with the Raman laser. These considerations make possible the extrapolation from ground performances to the needs for SAGAS. Main sources of systematic error are: magnetic field effects, one- and two-photon light shifts, laser wavefront distortions, $\mathrm{S} / \mathrm{C}$ vibrations and self-gravity.

The main limiting effect on accuracy comes from laser wave-front distortions. Although the effect can be calibrated in situ by changing the interaction time and/or the temperature of the atomic sample, more detailed ground studies should be carried out to confirm that the effect can be controlled at the required $5 \times 10^{-12} \mathrm{~m} \mathrm{~s}^{-2}$ level.

\subsubsection{Use as a micro-wave clock}

As all the elements needed to realize a micro-wave atomic clock at moderate accuracy $\left(10^{-12}\right.$ in relative frequency) are present in the payload, one can measure periodically the drift of the reference quartz oscillator, which is used to generate all radio or micro-wave frequencies for the accelerometer, the optical clock, the optical link and the on-board time scale. The atomic source and detection system are the same as for the accelerometer and the interrogation may be done by the micro-wave antenna used for the state preparation. Sensitivity of $10^{-12}$ per shot and the required $10^{-12}$ accuracy can easily be achieved.

\subsubsection{Description of the payload}

Most of the subsystems and components are similar or can be derived from the PHARAO project [1]. Estimations of mass and power budget take advantage of these similarities. The atomic accelerometer is composed of four subsystems (see Fig. 2): the vacuum tube where the atoms are cooled and interrogated (essentially the capture-zone sub-system of PHARAO), the optical bench for 
Fig. 2 Atomic accelerometer subsystems

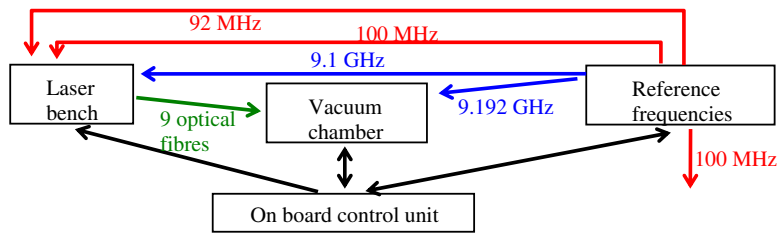

cooling and Raman transitions, the frequency reference for state preparation and Raman reference and the on board management unit to control the complete sequence and the acquisition.

The PHARAO heritage allows a relatively precise estimation of the mass, power and size budgets, as shown in Table 3.

\subsubsection{Current heritage and technology readiness level (TRL)}

Demonstrations of DC accelerometer on the vertical axis (gravimeter at $\leq 10^{-8} \mathrm{~m} \mathrm{~s}^{-2}$ ), limited by the presence of gravity and vibrations, have been done on ground, thus validating the method. The technology used in the payload is the same as PHARAO in the ACES project: same lasers, similar vacuum chamber, same frequency references. The engineering model of PHARAO passed all tests, which implies a high level of technology readiness also for the SAGAS accelerometer.

\subsection{Optical trapped ion clock}

The clock component of the overall mission scenario is based on the use of an on-board optical frequency standard with frequency stability $\leq 10^{-17}$ for 10 day integration times (i.e. an Allan variance of $\sigma_{y}(\tau) \leq 1 \times 10^{-14} \tau^{-1 / 2}$ ), where clock frequency data is downlinked to Earth by means of a high power link laser. This required performance is one order of magnitude better than the PHARAO clock and requires the development of a new generation of space clocks. Instead of the microwave frequencies used in PHARAO, the SAGAS clock uses optical frequencies. Ground based optical clocks are evolving at a significantly fast rate, with one or two single ion clocks already demonstrating instabilities $\sim 4 \times 10^{-17}$ at $<10^{4} \mathrm{~s}$ and $2 \times 10^{-17}$ accuracy in realising the unperturbed ion frequency [37-41]. There is good reason to expect similar performance for a range of other optical clock systems, which is extendable to

Table 3 Cold atom accelerometer mass, power, volume budgets

\begin{tabular}{lccl}
\hline Subsystem & Mass/kg & Power/W & Dimensions $/ \mathrm{mm}$ \\
\hline Vacuum tube & 21 & 5 & $400 \times 400 \times 500$ \\
Optical bench + electronics & 20 & 25 & $500 \times 300 \times 200$ \\
Reference frequencies & 7 & 12 & $300 \times 300 \times 100$ \\
On board unit control & 6 & 26 & $250 \times 250 \times 120$ \\
Total & 54 & 68 & 1251 \\
\hline
\end{tabular}


below the target specification at longer times. The range of options available to satisfy these stability/accuracy requirements is briefly outlined below.

\subsubsection{Choices for optical clocks}

Currently, there are two distinct high accuracy optical atomic clock architectures, one based on a single cold trapped ion approach, and one on cold neutral atoms held within an optical lattice. The single ion architecture presently represents a higher technology readiness level than is the case with neutral atom lattice clocks. In addition neutral atom clocks exhibit larger mass, volume and power consumption when compared to the ion clock arrangement. As a result, only the single ion clock is considered for this SAGAS proposal.

Within this ion clock arena, there are a number of possible ion species and isotopes which have been developed with state-of-the art characteristics: ${ }^{199} \mathrm{Hg}^{+},{ }^{27} \mathrm{Al}^{+},{ }^{115} \mathrm{In}^{+},{ }^{40} \mathrm{Ca}^{+},{ }^{171} \mathrm{Yb}^{+}$quadrupole, ${ }^{171} \mathrm{Yb}^{+}$octupole, ${ }^{88} \mathrm{Sr}^{+}$. For all species the quantum-limited theoretical stabilities surpass the stability requirement of $10^{-17}$ at 10 days. The experimentally observed stabilities are typically a factor 2 to 5 above this limit, but still within the $10^{-17}$ at 10 days specification.

Technological complexity in a space environment (generation of deep UV wavelengths, trap technology, UV optics and fibres, alignment tolerances,...) excludes a number of the above mentioned species, leaving three possibilities: ${ }^{40} \mathrm{Ca}^{+}(729 \mathrm{~nm}),{ }^{171} \mathrm{Yb}^{+}$quadrupole $(436 \mathrm{~nm})$, and ${ }^{88} \mathrm{Sr}^{+}(674 \mathrm{~nm})$. These show little difference in sensitivity to perturbing effects, essentially the effect of blackbody radiation and electric fields from the trap. As a result the choice is based on the availability of the required laser technology and the present state of ground systems. Taking together the currently available power levels for both clock and cooler, the need for only one single pass doubling stage, clock laser linewidth and the clock accuracy already achieved, it is considered that the ${ }^{88} \mathrm{Sr}^{+} 674 \mathrm{~nm}$ clock represents the most feasible ion clock option at this time. It is acknowledged that the technology underpinning all three options has the capability to evolve in the near to medium term, and this should be a core component of technology refinement activity during early stages of the L class mission preparations.

\subsection{2 ${ }^{88} \mathrm{Sr}^{+}$ion clock system}

The ion clock system design is outlined in Fig. 3. It comprises a number of sub-components, including:

- The primary physics package, an RF end-cap trap for ionising and confining a single ${ }^{88} \mathrm{Sr}{ }^{+}$ion within an ultra-high vacuum chamber pumped by a small ion pump and non-evaporable getter pump

- A laser platform providing Doppler cooling of the ion and auxiliary lasers for repumping and clear-out 


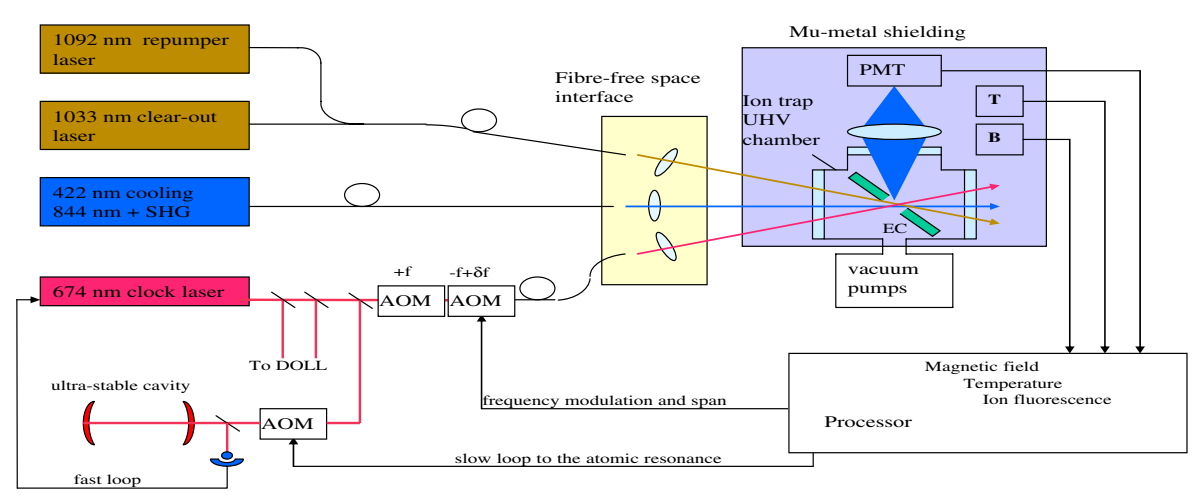

Fig. $3{ }^{88} \mathrm{Sr}^{+}$ion clock system architecture: AOM, acousto-optic frequency shifter; $P M T$, photomultiplier; $S H G$, second harmonic generation; $E C$, end-cap trap; $T$, temperature sensor; $B$, magnetic field sensor. Redundant laser units not shown, nor control lines for active unit monitoring and redundant unit activation after major fault diagnosis, nor standard monitoring and control lines

- The $674 \mathrm{~nm}$ clock laser frequency FM-stabilised to a very high finesse ultra-low-expansion (ULE) cavity mounted on a temperature-stabilised and evacuated platform. The clock laser is directly linked to the laser link (DOLL), see "Section 3.3"

- A monitoring and control processor driving the clock sequence (coolingprobing-detection). The processor also monitors frequency and amplitude data necessary to determine normal laser and ion operational conditions and initiate resetting and recovery algorithms where necessary, and laser unit failure.

- A redundancy level of two or three units for both cooling, clock and high power link laser, plus a redundancy level of three units for the repumper and clear-out DFB lasers. All redundancy units for each wavelength to be fibre multiplexed as standard, allowing redundant unit activation on determination of prior unit failure mode.

\subsection{3 ${ }^{88} \mathrm{Sr}^{+}$ion clock performance and critical issues}

The progress in the accuracy of single ion optical clocks has been rapid and it is foreseeable that within the next few years the level of one part in $10^{17}$ will be demonstrated in several ground based systems, including $\mathrm{Sr}^{+}$. The ${ }^{88} \mathrm{Sr}^{+}$ion clock systematic frequency dependencies are shown in Table 4, in the form of the expected uncertainty budget. Overall uncertainty of $\sim 10^{-17}$ is achievable within the spacecraft environment, provided external magnetic field and temperature variations are sufficiently low or adequately controlled.

Reloading of the trap will be necessary over a mission period of 15 years. A miniature dispenser, containing a few $\mathrm{mg}$ of $\mathrm{Sr}$ and a hot wire as an electron source are needed. Automatic reloading of the trap will induce an interruption 


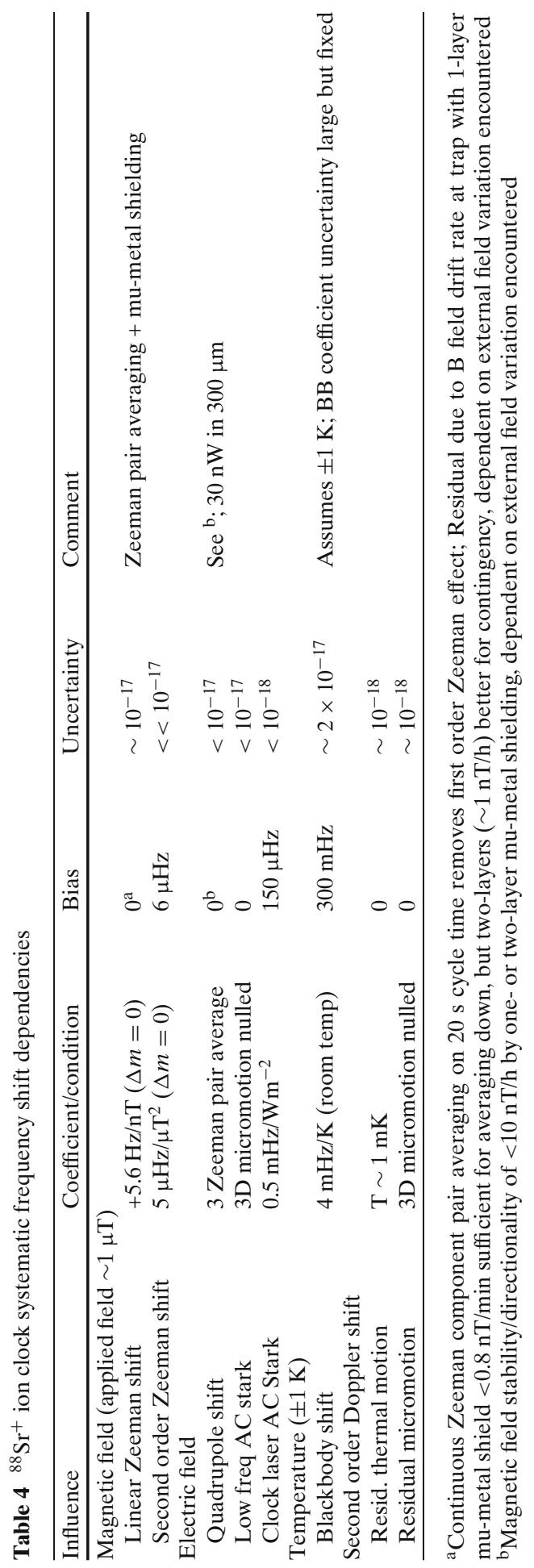


Table 5 Projected space ion clock power, volume and mass

\begin{tabular}{lllllll}
\hline $\begin{array}{l}\text { Type of } \\
\text { optical clock }\end{array}$ & Power (W) & $\begin{array}{l}\text { Physics } \\
\text { volume }\end{array}$ & $\begin{array}{l}\text { Electronics } \\
\text { volume }\end{array}$ & $\begin{array}{l}\text { Physics } \\
\text { mass }\end{array}$ & $\begin{array}{l}\text { Electronics } \\
\text { mass }\end{array}$ & $\begin{array}{l}\text { Total } \\
\text { mass }\end{array}$ \\
\hline Ion clock & 80 & 1501 & 301 & $50 \mathrm{~kg}$ & $30 \mathrm{~kg}$ & $80 \mathrm{~kg}$ \\
\hline
\end{tabular}

of clock operation for a few minutes. Charging problems of the trap and vacuum system will be avoided by illumination with ultraviolet LEDs. The discharge procedure will also be used periodically to eliminate charges from high energy radiation.

\subsubsection{Clock payload requirements}

Payload requirements are developed by extrapolation from existing groundbased clock arrangements, together with attention to existing laser and opto-electronic hardware and reference to the existing ACES/PHARAO microwave clock payload (Table 5). This includes a redundancy level of three lasers for both the clock laser ECDL and the high power $844 \mathrm{~nm}$ cooling laser and single pass doubler. The use of available DFB lasers for the 1,092 nm repumper laser and 1,033 $\mathrm{nm}$ clear-out laser will also allow a redundancy level of at least three per wavelength.

The physics package volume of $150 \mathrm{l}$ is considered an upper limit. It will include all laser systems, opto-electronic beam conditioning and fibre launching and delivery to the trap package, beam manipulation onto the ion, photomultiplier detection of the ion fluorescence, trap vacuum chamber and $21 \mathrm{~s}^{-1}$ ion pump plus non-evaporable getter pump, three-axis magnetic field coils for field nulling and quantisation axis definition, plus mu-metal shielding. The clock laser system will include one high finesse supercavity maintained within a small vacuum housing and pumped by a mini ion pump. The cooling/auxiliary lasers will require lower finesse smaller reference cavities for controlled wavelength tuning and stabilisation.

\subsection{Deep space optical laser link}

We propose an original optical link concept for SAGAS that takes full advantage of the particular technology available on-board and the synergy between payload components (narrow, stable and accurate laser from the clock, accurate microwave from the accelerometer) whilst being specially tailored to achieve the required science objectives. Concerning the technology, particular emphasis was paid to making maximum use of existing developments (ACES/PHARAO diode laser technology, LISA telescope technology, SLR/LLR ground stations,). DOLL features in particular:

- Continuous wave laser operation in both directions (two-way system)

- Heterodyne detection schemes on-board and on ground

- High data transfer rate with simultaneous science measurements 
- Asynchronous operation allowing optimal combination of on-board and ground measurements

- Large stray light rejection from heterodyne detection and due to the possibility of a large controlled frequency offset between the up and down link using the accurate on-board microwave

More generally, we believe that DOLL will not only find its use in SAGAS, but more generally contribute significantly toward the development of optical, high accuracy interplanetary navigation and broadband communication.

\subsubsection{Principle of operation and estimated performance}

DOLL is based on continuous two-way laser signals exchanged between the ground station and the $\mathrm{S} / \mathrm{C}$, with independent heterodyne detection of the incoming signal at either end (no transponder scheme). The fundamental measurement is the frequency difference between the local oscillator and the incoming signal. This measurement is particularly adapted to SAGAS because of the availability of the very narrow clock laser $(\approx 10 \mathrm{~Hz}$ linewidth $)$ on board and on the ground, stabilized to the atomic transitions with a stability of $\sigma_{\mathrm{y}}(\tau)=1 \times 10^{-14} \tau^{-1 / 2}$. The on-board and ground data are combined in post treatment and analysed in order to extract the science observables: Doppler $(\rightarrow$ velocity difference), clock frequency difference, ranging.

This allows asynchronous operation, i.e. the combination of ground and onboard measurements (sum or difference) with a delay that can be freely chosen thereby optimising the signal to noise ratio individually for any given science objective (see [3] for details).

To avoid complexity onboard the spacecraft, the signals emitted on the ground will be offset in frequency to largely compensate for the Doppler frequency shift (up to $70 \mathrm{GHz}$ ), so the frequency received at the $\mathrm{S} / \mathrm{C}$ is close to the nominal clock frequency, allowing direct heterodyne beat with the local clock laser. Additionally, the up and down signals will be linearly polarized with orthogonal polarizations (for stray light rejection), which implies variable polarization direction for the ground station telescope.

Data is encoded onto the laser signals of the up and down link using FSK (frequency shift keying), by modulating the laser frequency via the reference microwave signal frequency or PSK (phase shift keying) by modulating the phase of the reference microwave signal. Data is decoded by using a tracking oscillator phase locked on the incoming signal. The corresponding achievable data rate is about $3,000 \mathrm{bps}$ at $30 \mathrm{AU}$ with a negligible probability of error bits or cycle slips. The encoded signals also allow rough ranging and timing at the $\approx 3 \mathrm{~m}(10 \mathrm{~ns})$ level.

Note that for orbit determination the Doppler and ranging observables are, in principle, redundant. For SAGAS the precise orbit is obtained from the Doppler, whilst the ranging (orders of magnitude less precise) serves only to improve initial conditions for the integration. 


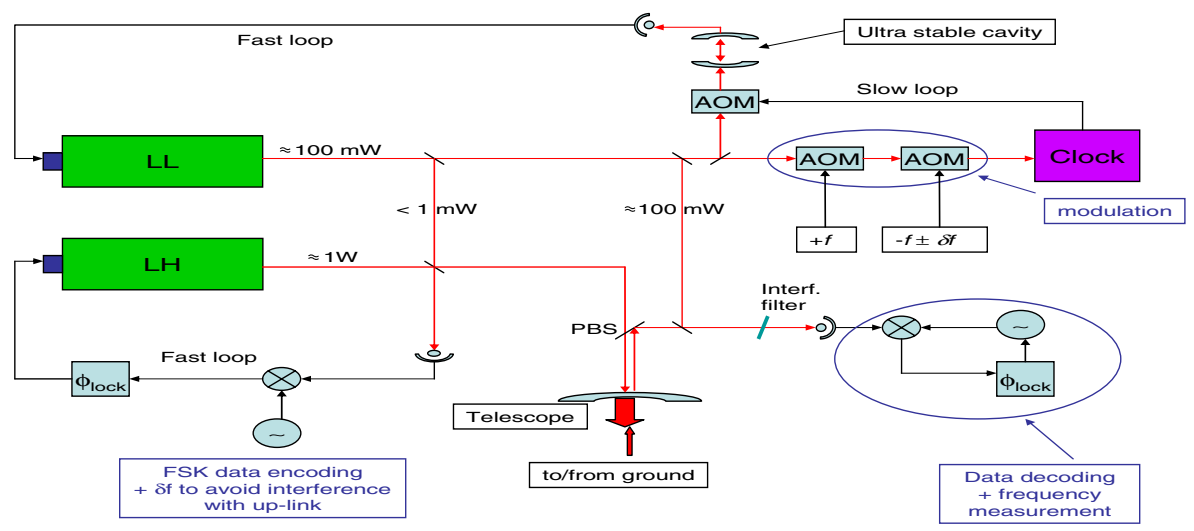

Fig. 4 Principle of the laser set-up for DOLL

\subsubsection{Space segment}

The main subsystems of the DOLL space segment are the telescope and the optical bench providing the laser source. The present baseline is to use a telescope design similar to that of the LISA mission ( $40 \mathrm{~cm}$ aperture), adapted for the SAGAS wavelength $(674 \mathrm{~nm})$ and including a high definition CCD camera (similar to COROT or LISA).

The optical bench houses a high power laser (LH) for the link, a low power laser (LL) for the clock, and an ultra stable cavity for short term laser stability ( $\approx 10 \mathrm{~Hz}$ linewidth), the long term stability being achieved by locking to the atoms in the optical clock (see Fig. 4). Several servo loops are used for locking of the lasers to each other, to the cavity, and to the atoms. Two acousto-optic modulators (AOM) serve for the modulation of LL to interrogate the atomic resonance. The corresponding error signal is sent to the third AOM thereby locking LL to the atoms. A fast phase locked loop (PLL) is used to lock LH to LL and to add an offset frequency $\delta f$ between the two (to mitigate stray light) and for the FSK (or PSK) modulation for data transmission. Another PLL serves for the heterodyne detection of the beat between the incoming signal and LL, which provides the science data (frequency difference) and gives access to the data modulated onto the frequency of the incoming signal.

\subsubsection{Ground segment}

The ground segment for DOLL will consist of several (three to six, with a minimum of three to ensure permanent coverage) laser tracking stations. Present satellite and lunar laser ranging stations are well adapted for this purpose, but will require upgrades concerning the optical set-up (wavelength, polarization control, pointing accuracy, adaptive optics) and need to be equipped with high performance optical clocks (see also "Section 5.3"). Also, choosing locations at high altitude with good seeing conditions is of importance to reduce the 
requirement on the adaptive optics system and reduce the probability of loosing lock due to atmospheric phase and intensity fluctuations.

\subsubsection{Error sources}

The main error sources affecting the performance of DOLL are related to power and pointing issues, stray light and effects of the Earth's atmosphere.

Assuming $1 \mathrm{~W}$ at emission, at a distance of $30 \mathrm{AU}$, a total attenuation of $\approx 0.14$ from losses in the atmosphere, the telescope, and from a pointing error of 0.3 " leaves about 22,000 photons per second at the photodiode, which corresponds to negligible shot noise of the heterodyne detection.

Stray light issues are strongly reduced by the narrow filter of the heterodyne detection PLL $(\approx 1 \mathrm{kHz})$. Particular care has to be taken to reduce in-band stray light from the outgoing signal. This is achieved by offsetting the outgoing frequency by about $\delta f=1$ to $10 \mathrm{GHz}$ using a PLL driven by the local RF signal.

The main limiting effect on the performance of DOLL will be fluctuations due to the Earth's atmosphere. At high frequency (around the $1 \mathrm{kHz}$ of the detection PLL) these can lead to frequent cycle slips and/or loss of lock. At low frequency atmospheric effects lead to phase fluctuations that can affect directly the observables and corresponding science measurements.

High frequency fluctuations are due to atmospheric turbulence and have been measured using optical stellar interferometry [42]. The results are reported in terms of the path delay structure function defined as $D(\tau) \equiv$ $\left\langle[x(t+\tau)-x(\mathrm{t})]^{2}\right\rangle$ with " $\langle$.$\rangle " denoting an ensemble average, and x$ the atmospheric optical path delay. For $\tau$ between $10 \mathrm{~ms}$ and a few seconds the observed $D(\tau)$ follows a power law with a slope around 1.5. At 10 ms typical values are $D(10 \mathrm{~ms}) \approx 5 \times 10^{-14} \mathrm{~m}^{2}$ [42]. This corresponds to fluctuations with an amplitude of about a third of the $674 \mathrm{~nm}$ wavelength of DOLL at $10 \mathrm{~ms}$ and to about $6 \%$ of the wavelength at $1 \mathrm{~ms}$. Thus cycle slips at the $1 \mathrm{kHz}$ bandwidth of DOLL are unlikely, but cannot be fully excluded, in particular during periods of large turbulence, wind velocities etc... Furthermore, intensity fluctuations, although mitigated by the adaptive optics and appropriate electronics (logarithmic amplifiers) can further increase the likelihood of loosing lock.

At longer integration times fluctuations are dominated by white phase noise due to turbulence, and slowly varying effects due to the evolution of global parameters (temperature, pressure, humidity). The structure function $D(\tau)$ undergoes a transition to white noise $(D(\tau) \approx$ const.) above a characteristic scale. In the results of [42] this transition occurs between $\tau=10 \mathrm{~s}$ and $\tau=100 \mathrm{~s}$ with $D(\tau)$ reaching a plateau at about $4 \times 10^{-10} \mathrm{~m}^{2}$. With a data rate of $0.01 \mathrm{~Hz}$ for DOLL this corresponds to a variance of $2 \times 10^{-10} \mathrm{~m}^{2}$ of the fluctuations of DOLL data. Additionally, we allow for slow variations, roughly linear over each individual $6 \mathrm{~h}$ continuous observation, with an amplitude of $1 \mathrm{~mm}$, corresponding to typical modelling errors of the tropospheric delay from global parameters as used in SLR [43, 44], and a roughly linear evolution of those parameters over $6 \mathrm{~h}$. This corresponds to a white frequency noise with a variance of about $2.4 \times 10^{-32}$ in relative frequency at $6 \mathrm{~h}$ averaging. 
Table 6 DOLL budgets

\begin{tabular}{lllll}
\hline & LH & LL & ULE cavity & $\begin{array}{l}\text { AOM + RF } \\
+ \text { electronics }\end{array}$ \\
\hline Power (W) & 25 & 3 & 3 & 20 \\
Mass & $89 \mathrm{~kg}$ & & & \\
Volume & 301 (not including telescope) & \\
\hline
\end{tabular}

To model the corresponding overall atmospheric noise, we generate data (100 s points) with a white phase noise corresponding to $210^{-10} \mathrm{~m}^{2}$ delay fluctuations, form the derivative, and then add $6 \mathrm{~h}$ constants randomly distributed with $2.4 \times 10^{-32}$ variance in relative frequency. The resulting PSD in relative frequency corresponds to $S_{\mathrm{y}}(f) \approx 1.8 \times 10^{-23} f^{2}$ per Hertz for $f>3 \times 10^{-4} \mathrm{~Hz}$ corresponding to the white phase noise from turbulence. It then increases with decreasing frequency reaching a plateau at $S_{\mathrm{y}}(f) \approx 1 \times 10^{-27}$ per Hertz for $f<3 \times 10^{-5} \mathrm{~Hz}$ corresponding to the added white frequency noise.

\subsubsection{Power, mass, volume}

The DOLL power and mass are estimated based on the PHARAO clock (diode lasers, AOM, electronics, RF source) and LISA studies (telescope, optical components etc...). Note also that some of the subsystems (LL, ultra stable cavity, RF source) are already accounted for in the clock or accelerometer budgets, but are included also here for additional margin and to reflect the early stage of the design (Table 6).

\subsubsection{Critical issues, requirements, heritage, and technology development}

The main DOLL critical issues are the 0.3" pointing requirement and the laser reliability. They are discussed in more detail in "Sections 4.3" and "Section 6.2" respectively. Much of the DOLL technology is based on heritage from PHARAO (ECDL, AOM, RF synthesis, Quartz,...), COROT (CCD, pointing) and on LISA technology developments (telescope, optics,...). That ensures a relatively high TRL (Technology Readiness Level) for most of the sub-systems, which is however contingent on the reliability and the development of the high power laser source at $674 \mathrm{~nm}$.

Finally, we note, that the development of DOLL as well as the optical clocks for the DOLL ground stations fits well into current technology drives towards the use of optical clocks and optical communication in NASA and ESA interplanetary missions and DSN.

\section{Spacecraft key factors}

SAGAS has the challenging task to measure the gravitational field in the Solar System between 1 and 50 AU. The preliminary concept satisfying the requirements put forward by its tasks consists of a three-axis stabilised spacecraft 
with excellent pointing accuracy. The power demand of the order of $400 \mathrm{~W}$ is fed by two Radioisotope Thermoelectric Generators (RTGs). A bi-propellant propulsion module (PM) is attached to SAGAS that serves to increase the Earth escape velocity and/or to conduct required deep space manoeuvres (see "Section 5").

\subsection{Design drivers}

The design drivers for SAGAS arise mainly from the needs of the payload and the requirements during a long journey to the outer Solar System.

A general requirement for the payload of SAGAS is high thermal stability, because the precision instruments will deliver best performance in a stable environment. Specific requirements for the cold atom accelerometer and the interplanetary laser link come on top of this:

- The cold atom sensor has the same need as any conventional precision accelerometer to be placed precisely in the centre of mass of the spacecraft with as little self gravity gradient from the spacecraft in its surroundings as possible.

- The laser link is a two-way asynchronous connection. The narrow beam divergence implies a pointing requirement of 0.3" (see "Section 3.3"). As a further complication the uplink and downlink will have to point into different directions due to the long two-way light time.

The journey to the outer Solar System puts forward the following requirements to the system:

- A power supply that is still functional at low insulation and that satisfies the considerable power demands of the SAGAS payload of about $200 \mathrm{~W}$

- A robust communication system suited for the long distance as a backup to the laser communication system

- A thermal control that can cope with a factor 2,500 change in impinging solar power.

In the following sections the solutions for each of the drivers, identified above, are addressed in the context of the relevant subsystem.

\subsection{Configuration}

The SAGAS spacecraft is a three-axis stabilised platform (Fig. 5). One side is permanently pointing towards Earth. This side carries both the high gain antenna (HGA) and the Telescope for the laser link. The opposite panel of the $\mathrm{S} / \mathrm{C}$ carries the adapter ring towards the propulsion module. Two RTG power supplies are placed on the side panels on short struts. The length of the struts is limited by the space in the fairing and by the launch loads. The longest possible struts for the selected launcher should be chosen in order to minimise the view factor of the RTGs towards the S/C side panels. This serves to minimise the backscattering of thermal radiation from the spacecraft 


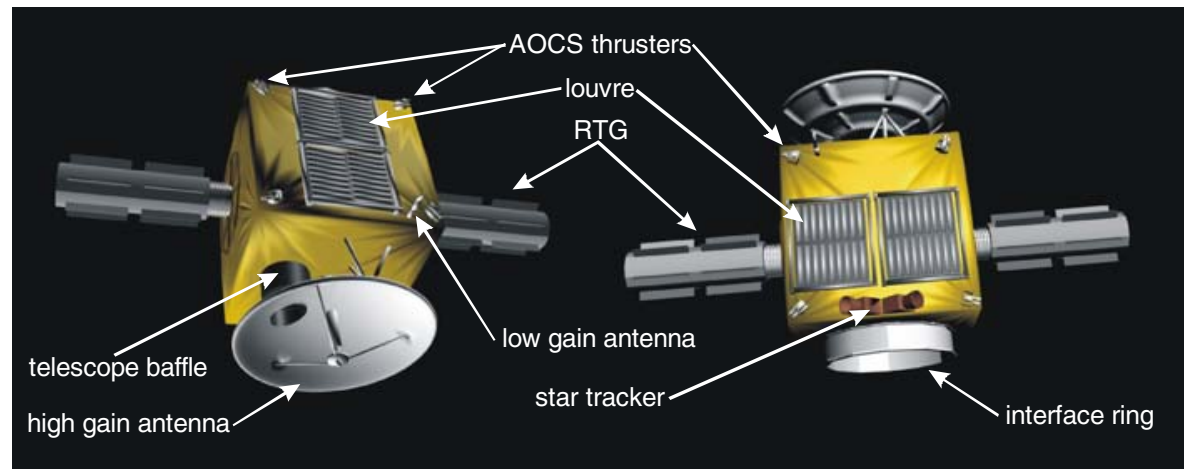

Fig. 5 Configuration concept of the SAGAS spacecraft

hull that contributes an unwanted source of acceleration systematics. For the interior configuration care has to be taken in order to minimize the magnitude of centre of mass movements. For this reason the hydrazine monopropellant for AOCS is distributed over three tanks that are placed towards the sides of the bus and symmetrically with respect to the centre of mass.

\subsection{AOCS}

The SAGAS spacecraft needs to be three-axis stabilized in order to fulfil the pointing needs of the laser link. The attitude control system consists of sensors and actuators. A Sun sensor is foreseen for initial attitude acquisition in LEOP and safe mode. Star trackers and an inertial measurement unit are used for attitude determination for coarse pointing, e.g. during trajectory manoeuvres. During science mode the attitude determination is supported by a feedback from the detector of the uplink laser signal. This detector is designed as a quadrant photodiode and can hence provide fine pointing information with respect to the uplink direction by differential wave front sensing. The same feedback from the photodiodes to the AOCS is foreseen for LISA.

Standard reaction wheels of $12 \mathrm{Nm}$ capacity and a set of 12 (+12 redundant) $10 \mathrm{~N}$ thrusters are foreseen as actuators. Wheels of a sufficient lifetime for SAGAS are available for instance from Rockwell Collins, Germany. In all nominal modes the attitude control of SAGAS relies on reaction wheels. The fine-pointing at the required level using conventional reaction wheels and a feedback loop from the payload is currently being demonstrated by the COROT spacecraft and is hence also foreseen for SAGAS. It was found that the low frequency vibrations induced by the reaction wheels are compatible with the accelerometer and optical cavity vibration requirements.

A particular challenge for the AOCS, and indeed SAGAS as a whole, is maintaining lock to the incoming laser, and initial acquisition of that lock. The solution to this problem lies in the combined use of the CCD (FoV of 1') that the $\mathrm{S} / \mathrm{C}$ telescope is equipped with, and the quadrant diode $(0.3$ " FoV) used for 
the heterodyne detection (a combination of the COROT and LISA methods for fine pointing). Initial acquisition is achieved in the following way:

1. The $\mathrm{S} / \mathrm{C}$ establishes the right pointing towards a ground station $\mathrm{G}$ with respect to the Earth's image in the CCD using onboard information about the station position at a given time with respect to the Earth's limb and starts emitting, applying the necessary point ahead angle so that the signal arrives at $\mathrm{G}$ (or another) ground station (see below for requirements).

2. The Gs all stare with their CCDs (large FoV) waiting for an incoming signal. On reception they lock onto it, apply the necessary point ahead angle and emit back.

3. The $\mathrm{S} / \mathrm{C}$ thus receives a signal back, already in its quadrant diode FoV, and locks onto it.

Note, that lock remains established even when Gs change due to Earth's rotation because the S/C "knows" where the different Gs are, and thus switches from one to the next as required (once lock is established the $\mathrm{S} / \mathrm{C}$ disposes of the incoming laser additionally to the CCD image when switching Gs). It also switches the point ahead angle (not synchronously with the reception switch, but offset by the light travel time) so Gs always have an arriving laser to lock to (they do not need to scan for the S/C with their CCD). The procedure requires that the $\mathrm{CCD}$ FoV and the quadrant diode FoV be aligned to better than 0.3". LISA studies have shown that alignment to below 0.2 " is possible. For SAGAS, additionally, we have the possibility of post launch calibration of that alignment using the large CCD image of the Earth and large incoming laser power in the early mission phase. Secondly, the CCD needs to be able to resolve a target on the Earth image corresponding to the 0.3 " diode FoV, i.e. about $200 \mathrm{~km}$ at $1 \mathrm{AU}$ and 6,500 $\mathrm{km}$ at $30 \mathrm{AU}$, which should be possible given COROT performance.

\subsection{Power subsystem}

For a mission to several tens of AU a radio thermal power supply is mandatory. The payload and system power demands are $400 \mathrm{~W}$ in total, both in cruise mode with PM and in science mode. For the preliminary design, four ASRG RTGs which are currently being developed by Lockheed-Martin in the US are considered. The development plan by NASA and the US Department of Energy for RTGs foresees that these Stirling Radioisotope Generators will be available in 2009 and hence well in time for the Cosmic Vision timeframe. The performance assumptions for the ASRG are based on [45]. The specific type of RTGs is not important for SAGAS. For instance, a set of two current US GPHS RTGs would also be a suitable choice that fulfils the power and lifetime requirements of SAGAS. 


\subsection{Radio-frequency telecommunication system}

An X-band communication system is foreseen for telemetry tracking and command. The radio link is mandatory because during critical mission phases such as orbit manoeuvre and in safe mode the laser link will not be available. For LEOP two low-gain antennas with hemispherical coverage are foreseen. For deep space communications a high-gain antenna (HGA) is baselined. It is assumed that the communication can be established via the HGA also in safe mode relying only on Sun-sensor information using a conscan manoeuvre. Hence currently a medium gain antenna is not foreseen. Since the current design is not mass critical this decision may be revisited. The accommodation of the HGA is constrained by the accommodation of the $40 \mathrm{~cm}$ aperture telescope. Two options were identified, a $1.1 \mathrm{~m}$ diameter HGA placed side by side with the telescope or a $2.2 \mathrm{~m}$ diameter antenna with a cut-out for the telescope. Both options could provide sufficient data rates (50 and 180 bps respectively at the end of mission) taking into account that the channel for the science data and regular telemetry is via the laser link.

The larger antenna with the cut-out is the currently preferred solution due to its higher performance. A detailed assessment of the effect of the cut-out on the antenna pattern is however needed to finalize the decision.

\section{Mission profile}

The objective of the SAGAS trajectory is to reach the end of mission target, a heliocentric distance of $50 \mathrm{AU}$ in as short time as possible. The total mission duration is 15 years nominal and 20 years extended mission. The spacecraft wet mass under consideration will be $1,000 \mathrm{~kg}$. The trajectory foresees a Jupiter gravity assist to reach hyperbolic escape velocity. Depending on the chosen launcher, one or more gravity assists in the inner Solar System may be required to reach Jupiter.

\subsection{Escape strategy and launcher selection}

Combined with the sizeable spacecraft mass, a large launcher is the only realistic option. The only currently available European launcher that falls into this category is the Ariane 5 ECA. For a $671 \mathrm{~kg}$ spacecraft, it offers a hyperbolic excess velocity of $7 \mathrm{~km} / \mathrm{s}$ [46]. Unfortunately, this performance is too low to put a spacecraft of the desired mass into a trajectory towards Jupiter. Hence even with an Ariane 5 ECA, a gravity assist in the inner Solar System will be required to put the spacecraft on the desired trajectory.

The situation could considerably improve with the advent of the Ariane 5 $\mathrm{ECB}$, which will feature the re-ignitable Vinci upper stage engine. With the Ariane $5 \mathrm{ECB}$, a $1,000 \mathrm{~kg}$ spacecraft could reach an escape velocity of $9.5 \mathrm{~km} / \mathrm{s}$, 
Table 7 Launcher options for SAGAS

\begin{tabular}{ll}
\hline Launcher options & $\begin{array}{l}\text { Hyperbolic excess velocity }(\mathrm{km} / \mathrm{s}) \\
\text { for 1,000 kg payload }\end{array}$ \\
\hline Ariane 5 ECA & $\sim 6.5$ \\
Ariane 5 ECB & $\sim 9.5$ \\
Atlas V/Star 48 V & $\sim 10.7$ \\
Delta IV Heavy/Star 48B & $\sim 10.0$ \\
Proton M/Breeze M & $\sim 8.1$ \\
\hline
\end{tabular}

which would be more than sufficient for a direct transfer to Jupiter. It is likely that the Ariane 5 ECB will be available for the Cosmic Vision time frame.

Other currently available launcher options that would allow a direct transfer to Jupiter are listed in Table 7. These launchers could be considered if the mission is realised in collaboration with other space agencies.

For the Ariane 5 launcher the use of a propulsion module (PM) improves the escape performance, that could be either the US Star 48 B PM or a bipropellant PM based on the Eurostar platform and the LISAPathfinder PM that has been derived from it.

From this survey, it is clear that the Ariane $5 \mathrm{ECB}$, the American Atlas V and Delta IV and the Russian Proton allow a direct transfer to Jupiter if a kickstage or PM is used. For the Ariane 5 ECA, a direct transfer to Jupiter is not obtainable even with a PM.

\subsection{Trajectory design}

Amongst the launchers discussed above, only the Ariane 5 ECA is considered in the launcher list of the Cosmic Vision frame (ESA document D/SCI/DJS/SV/val/21851, Annex 4). Hence, we consider the Ariane 5 ECA as our preferred launcher option. Unfortunately, this launcher has the lowest performance, and hence requires a more complicated trajectory design to reach the desired heliocentric distance of $50 \mathrm{AU}$. The identified solution is an Earth-Earth-Jupiter trajectory with two gravity assists. In this option the spacecraft is first put into a resonant orbit with Earth of 2 or 1.5 years period and a sizeable deep space manoeuvre of $\sim 0.5 \mathrm{~km} / \mathrm{s}$ is conducted at aphelion to amplify the effect of the gravity assist at Earth. Such trajectories are commonly denoted as $\Delta \mathrm{V}$-EGA trajectories. Hyperbolic escape velocity is reached after Jupiter and the SAGAS spacecraft reaches 50 AU in a hyperbolic coast.

For preliminary mission analysis, a global optimisation has been carried out to determine the preferred transfer opportunity with a launch date between 2017 and 2025. The objective was to reach $50 \mathrm{AU}$ in minimal travel time under the combined constraints of the Ariane 5 ECA escape capability and the maximal $\Delta \mathrm{V}$ capability of the propulsion module for the respective escape conditions. The optimal trajectory with a departure date in the Cosmic Vision timeframe has a launch in March 2019 and leads to a transfer to 50 AU in only 18.83 years. The key parameters of the resulting optimal trajectory are given in Tables 8 and 9. The trajectory until Jupiter is depicted in Fig. 6. 


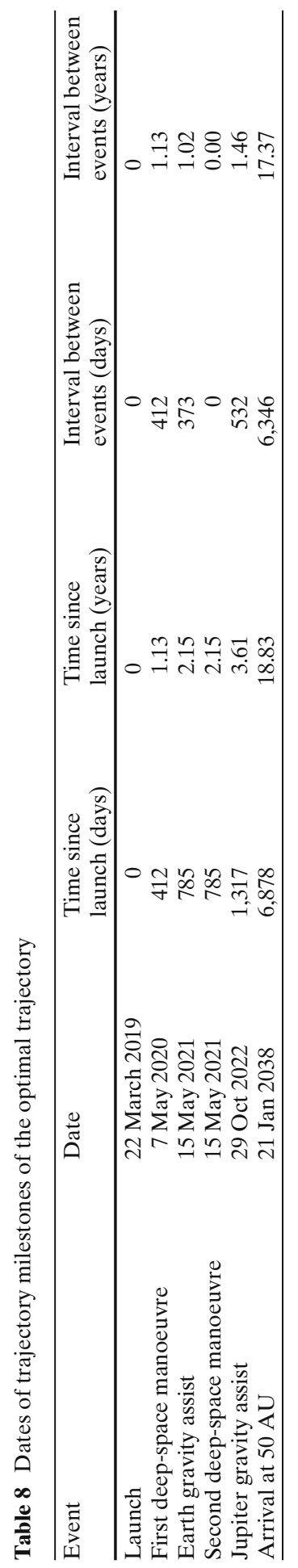


Table 9 Velocity parameters of the optimal trajectory

\begin{tabular}{lc}
\hline Parameter & Value \\
\hline Earth departure velocity $(\mathrm{km} / \mathrm{s})$ & 5.27 \\
First deep space $\Delta \mathrm{V}(\mathrm{km} / \mathrm{s})$ & 0.656 \\
Second deep space $\Delta \mathrm{V}(\mathrm{km} / \mathrm{s})$ & 0.848 \\
Total deep space $\Delta \mathrm{V}(\mathrm{km} / \mathrm{s})$ & 1.504 \\
Velocity after Jupiter swingby $(\mathrm{km} / \mathrm{s})$ & 22.64 \\
\hline
\end{tabular}

Generally, launch opportunities towards Jupiter open up once a year. The analysis showed that the typical travel times for these opportunities are between 19 and 21 years. Hence, allowing a 10\% longer travel time, the launch of SAGAS can take place in any desired year. This flexibility in launch date is a particular benefit of the $\Delta \mathrm{V}$-EGA for which only an optimal constellation of Earth and Jupiter is required which arises once a year.

For the other launchers in Table 7 a direct transfer to Jupiter is possible. This would allow to omit the EGA loop and hence to shorten the mission duration by approximately 2 years.

In summary, with the chosen trajectory SAGAS will reach a heliocentric distance of 38.9 AU over the nominal mission duration (15 years) and 53.3 AU over extended mission (20 years).

\subsection{Ground segment}

For the X-band link the mission will use ESA $15 \mathrm{~m}$ ground stations for LEOP operations and ESA $35 \mathrm{~m}$ ground stations for deep space communications. During most of the mission, ground contact will be infrequent-less than once a fortnight - because the science and telecommand data are transmitted

Fig. 6 Optimal $\Delta V$-EGA trajectory for SAGAS through the inner Solar System (red/black). Orbits of Earth and Jupiter in blue. Dimensions in kilometer

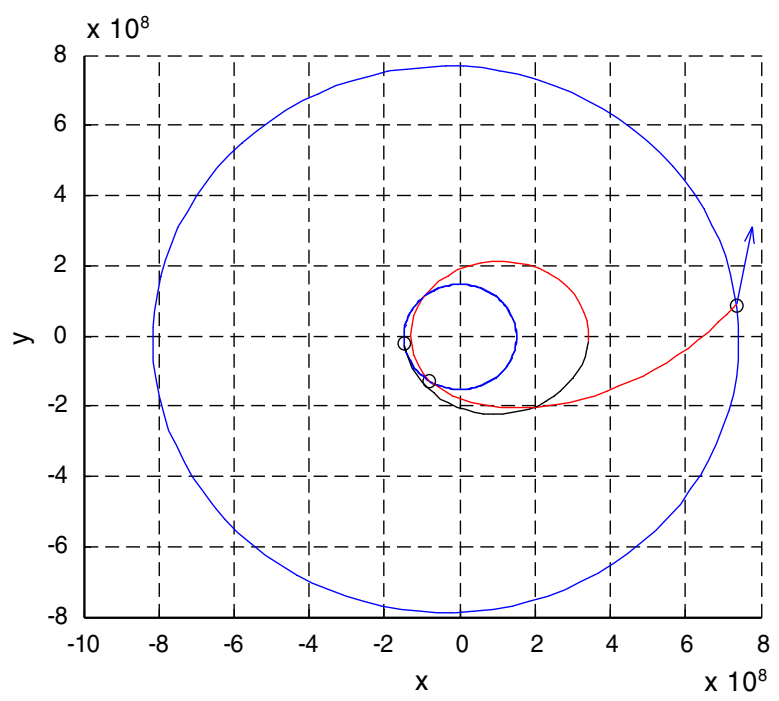


via the laser link (kbps capacity, see "Section 3.3"). For periods around the gravity assists and the deep space manoeuvres, permanent ground coverage via $\mathrm{X}$-band and laser is desirable. In general no large baseline tracking operations will be required because orbit reconstruction accuracy from the laser ranging will considerably exceed $\Delta$ VLBI performances.

The laser link will require three to six dedicated ground stations equipped with $1 \mathrm{~m}$ or larger telescopes (see "Section 3.3"), optical clocks and corresponding laser systems (see also "Section 6.2"). The corresponding observation program will require continuous observation of the satellite whilst visible, in particular during critical science phases (e.g. a few days before and after occultations for the PPN test). The present baseline for such ground stations is to take advantage of the existing structure of Satellite and Lunar Laser Ranging stations, several of which are already equipped with $1.5 \mathrm{~m}$ telescopes (OCA, Matera,...). The corresponding stations will require upgrades to make them compatible with SAGAS requirements; in particular, they will require adaptive optics, optical clocks and corresponding laser systems. An alternative option would be to develop dedicated laser DSN stations for SAGAS, but also for other deep space missions that will require precise timing, navigation and broadband communication. Both options fit well into the general technology development of laser communication and optical clocks for DSN, carried out presently under ESA and NASA contracts.

\section{Key technology areas}

\subsection{Platform}

The SAGAS spacecraft faces considerable challenges in terms of instrument accommodation, pointing requirements and the need to provide sufficient power in the outer Solar System. The preliminary concept ("Section 4") has addressed all theses challenges and has identified feasible solutions. The biggest challenge is the differential wave-front sensing for the locking of the laser beam. The corresponding technology has already been demonstrated for LISA in a laboratory setup. Nonetheless, while considerable experience can be drawn from COROT, GAIA and the technology development for LISA, a detailed analysis of the beam recovery and locking scheme for SAGAS is likely to reveal important differences. Hence a technology development activity on the attitude for SAGAS during science mode should be initiated timely.

The location requirement of the cold-atom accelerometer in close distance from the centre of mass is comparable to those of GOCE or LISA Pathfinder and hence ample experience on this task will exist in Europe for the implementation of SAGAS.

Otherwise, no particular technology development needs for the platform could be identified. The complete platform of SAGAS uses well developed technology and hence the challenges of SAGAS lie mainly in its payload and operations and not in the platform. Notable exceptions are the Stirling 
radioisotope generators, which are still under development. These are, however, not considered critical because the type of RTGs to be used is not critical for SAGAS and it can be assumed that an RTG of the power-to-mass ratio of the current GPHS RTGs will, in any case, be available in the US during the Cosmic Vision timeframe.

It is worth stressing that sufficient expertise for the power system design using RTGs and their integration exists in Europe: GPHS RTGs have already been used on the joint ESA/NASA mission Ulysses. For Ulysses, both the power system was designed and the RTGs were integrated by a European company. While the knowledge for the handling of RTG is available in Europe, no corresponding handling and safety regulations for Centre Spatial Guyanais (CSG) have been established, yet. First steps towards such regulations are currently undertaken for radioisotope heating units within ESA's Aurora programme. However, it is likely that the larger amount of nuclear material present in the RTGs will require additional regulations. Hence, the qualification of CSG for the launch of RTGs should be initiated well ahead of the SAGAS implementation phase. A possible way to circumvent this qualification would be the launch on a US launcher. This option was found attractive from the point of view of trajectory design as well (cf. "Section 5").

\subsection{Laser sources for clock and optical link at $674 \mathrm{~nm}$}

At $674 \mathrm{~nm}$ with the required $1 \mathrm{~W}$ output, diode laser technology requires the use of an ECDL followed by a tapered amplifier (TA). At present, the maximum output power available at $674 \mathrm{~nm}$ using ECDL-TA configuration is $250-300 \mathrm{~mW}$, but power upgrades are expected from the development of longer TPA chips. Output power exceeding $1 \mathrm{~W}$ is realistic within 3 years from now. Accelerated ageing tests have been performed with AR-coated $670 \mathrm{~nm}$ wavelength ECDLs $\left(90^{\circ} \mathrm{C}\right.$ operation temperature), with typical output powers of 10 to $20 \mathrm{~mW}$. An extrapolated value $>10^{4} \mathrm{~h}$ was found in standard operating conditions. No significant dependence of the lifetime on the optical power was found, so that this result can be representative also in case of higher optical power. In standard operating conditions $\left(25^{\circ} \mathrm{C}\right)$ lifetimes exceeding $2.5 \times 10^{4} \mathrm{~h}$ (about 3 years) have been already reported by different customers at $671 \mathrm{~nm}$ wavelength. Lifetimes approaching $10^{5} \mathrm{~h}$ are not unrealistic in the future (in fact such lifetime values have been already demonstrated in case of infrared laser diodes for telecom applications) but would require significant developments for the chips and 3-5 years from now. It is thus realistic to expect to be able to cover the complete mission duration with moderate (two or threefold) redundancy.

An alternative approach based on laser diode technology that could become realistic in the near future is a directly modulated DFB laser $(100 \mathrm{~mW}$ oscillator) or a DFB laser as master laser within a master oscillator power amplifier system (MOPA) in the case of larger optical power (up to $1 \mathrm{~W}$ ). DFB lasers offer a significant advantage in comparison with external cavity diode lasers in terms of dimensions and mode-hop free tuning range without 
any moving mechanical part. The lack of mechanical parts qualifies DFB lasers especially for space applications. Currently, this concept can be realized within the wavelength interval between 730 and $1,060 \mathrm{~nm}$, but further developments are expected in the next years to extend the emission wavelength towards the visible region.

Although operation of the optical link with $1 \mathrm{~W}$ ground lasers is certainly possible (symmetric to the down link), it will likely be of advantage to use higher power $(>10 \mathrm{~W})$ on the ground, if available. To that aim a realistic solution was identified using a coherent solid-state device at $674 \mathrm{~nm}$ based on intracavity frequency doubling of the ${ }^{4} \mathrm{~F}_{3 / 2} \rightarrow{ }^{4} \mathrm{I}_{13 / 2}$ laser transition at $1,348 \mathrm{~nm}$ of $\mathrm{Nd}^{3+}$ doped crystals. The proposed laser source is based on a slave laser with a thin disk pumping scheme, using a high Nd-doping concentration in a $\mathrm{La}_{2} \mathrm{SiO}_{5}$ crystal (in this case the La ionic radius closely matches the $\mathrm{Nd}$ one), injected by a tunable, single-frequency, narrow-linewidth oscillator $(500 \mathrm{~mW}$, linear cavity). Using this optical configuration we expect to obtain $30 \mathrm{~W}$ at $1,348 \mathrm{~nm}$ using $100 \mathrm{~W}$ pump power at $808 \mathrm{~nm}$ and $12-15 \mathrm{~W}$ output power at $674 \mathrm{~nm}$ by intracavity frequency doubling.

In summary, availability, space qualification, and lifetime of laser sources for the clock and the link $(674 \mathrm{~nm})$ is a key technological issue for SAGAS, with a presently low technology readiness. However, given the large technology basis in the field of semiconductor and solid state lasers a rapid development of appropriate laser sources seems likely but should be initiated early.

\section{Conclusion}

We have presented a detailed description of the scientific and technological aspects of the SAGAS project, based on the original mission proposal submitted to ESA in June 2007 in response to the Cosmic Vision 2015-2025 call for proposals. The outcome of the ESA selection procedure is now known, and unfortunately SAGAS has not been deemed a priority. However, independently of that outcome, we believe that it is worth pursuing the investigations initiated by the Cosmic Vision call, in order to further study the scientific and technological implications of this type of mission.

On the science side, deep space gravity probes are unique opportunities to address some of the most fundamental questions of contemporary physics, related to unification of the fundamental interactions in nature, the nature of gravitation, dark energy and dark matter. By extending experimental tests of gravity to the largest scales attainable by human-made artifacts (size of the Solar System), missions like SAGAS are starting to bridge the gap between observational evidence at relatively short scales ( $\approx$ Earth-Moon distance) where all observations confirm present theories, and astronomical (Galaxies) and cosmological scales where agreement between theory and observation comes at the expense of postulating large amounts of dark matter and energy.

Concerning the exploration of the outer Solar System (Kuiper belt, giant planets), SAGAS opens a new and complementary window on such 
exploration, no longer based on electromagnetic imaging, but on the measurement of the gravitational signatures of the objects to be studied or discovered. The determination of the Kuiper belt mass distribution and total mass are good examples where gravitational measurements are complementary to, and better adapted than "classical" techniques.

The SAGAS payload will include an optical atomic clock optimised for long term performance, an absolute accelerometer based on atom interferometry and a laser link for ranging, frequency comparison and communication. The complementary instruments will allow highly sensitive measurements of all aspects of gravitation via the different effects of gravity on clocks, light, and the free fall of test bodies, thus effectively providing a detailed gravitational map of the outer Solar System, whilst testing all aspects of gravitation theory to unprecedented levels. It is expected that similar instruments, and optical links in general, will find their way into future terrestrial and solar system missions, whenever precise knowledge of local gravity, precise timing, or precise navigation are required.

In summary, SAGAS opens the way towards the experimental investigation of some of the most puzzling questions of contemporary physics and towards a new window for the exploration of the outer Solar System. These "phenomena of the very large" are explored using the "technology of the very small", quantum sensors, illustrating the discovery potential of the combination of the two domains.

\section{References}

1. Laurent, P., et al.: Appl. Phys. B 84, 684-690 (2006). doi:10.1007/s00340-006-2396-6

2. Groten, E.: Report of the IAG. Special Commission SC3, Fundamental Constants, XXII IAG General Assembly (1999)

3. Reynaud, S., et al.: PRD 77, 122003 (2008). arXiv:0801.2896

4. Vessot, R.F.C., et al.: PRL 45, 2081 (1980). doi:10.1103/PhysRevLett.45.2081

5. Saathoff, G., et al.: PRL 91, 190403 (2003). doi:10.1103/PhysRevLett.91.190403

6. Hohensee, M., et al.: PRD 75, 049902 (2007). doi:10.1103/PhysRevD.75.049902

7. Will, C.M.: Living Rev. Relativity 9, (2006)

8. Bertotti, B., Iess, L., Tortora, P.: Nature 425, 374 (2003). doi:10.1038/nature01997

9. Anderson, J.D., et al.: PRL 81, 2858 (1998)

10. Turyshev, S.G., et al.: arXiv:gr-qc/990024 (1999)

11. Anderson, J.D., et al.: PRD 65, 082004 (2002)

12. Turyshev, S.G., et al.: Int. J. Mod. Phys. D15, 1 (2006)

13. Toth, V.T., Turyshev, S.G.: AIP Conf. Proc. 977, 264 (2008) arXiv:0710.2656

14. Lämmerzahl, C., Preuss, O., Dittus, H.: In: Dittus, H., Lämmerzahl, C., Turyshev, S.G. (Eds.) Lasers, Clocks, and Drag-Free: Exploration of Relativistic Gravity in Space, pp. 75. Springer, Heidelberg (2007)

15. Morbidellie, A.: The dynamical structure of the Kuiper Belt and its primordial origin. In: Baruuci, A., Boehnhardt, H., Cruikshank, D., Morbidelli, A. (eds.) The Kuiper Belt. Univ. of Arizona Press, Tucson (2007)

16. Bertolami, O., Vieira, P.: CQG 23, 4625 (2006). doi:10.1088/0264-9381/23/14/005

17. Bertolami, O., Páramos, J.: Int. J. Mod. Phys. D 16, 1611-1623 (2007). doi:10.1142/ S0218271807011000

18. Bernstein, G., et al.: ApJ. 128, 1364 (2004)

19. Uzan, J.-P.: Rev. Mod. Phys. 75, 403 (2003). doi:10.1103/RevModPhys.75.403 
20. Murphy, M.T., et al.: Mon. Not. R. A. S. 345, 609 (2003). doi:10.1046/j.1365-8711.2003.06970.x

21. Reinhold, E., et al.: PRL 96, 151101 (2006). doi:10.1103/PhysRevLett.96.151101

22. Flambaum, V.V., Shuryak, E.V.: arXiv: physics/0701220 (2007)

23. Damour, T., Nordtvedt, K.: PRL 70, 2217 (1993)

24. Damour, T., Nordtvedt, K.: PRD 48, 3436 (1993). doi:10.1103/PhysRevD.48.3436

25. Damour, T., Polyakov, A.M.: Nucl. Phys. B 423, 532 (1994) arXiv:hep-th/9401069. doi:10.1016/0550-3213(94)90143-0

26. Armstrong, J.W., et al.: ApJ. 318, 536 (1987). doi:10.1086/165390

27. Armstrong, J.W., et al.: ApJ. 599, 806 (2003). doi:10.1086/379505

28. Anderson, J.D., Mashoon, B.: ApJ. 290, 445 (1985). doi:10.1086/163002

29. Bordé, Ch.J.: Metrologia 39, 435 (2002)

30. Tino, G.M., et al.: Nucl. Phys. B Proc. Suppl. 166, 159 (2007). doi:10.1016/j.nuclphysbps. 2006.12.061

31. Peters, A., et al.: Metrologia 38, 25-61 (2001)

32. Canuel, B., et al.: Phys. Rev. Lett. 97, 010402 (2006). doi:10.1103/PhysRevLett.97.010402

33. Bertoldi, A., et al.: EPJD 40, 271-279 (2006)

34. Fixler, J.B., et al.: Science 315, 74 (2007). doi:10.1126/science.1135459

35. Wicht, A., et al.: Phys. Scr. T 102, 82 (2002). doi:10.1238/Physica.Topical.102a00082

36. Cladé, P., et al.: Phys. Rev. Lett. 96, 033001 (2006). doi:10.1103/PhysRevLett.96.033001

37. Fortier, T., et al.: PRL 98, 070801 (2007). doi:10.1103/PhysRevLett.98.070801

38. Oskay, W.H., et al.: PRL 97, 020801 (2006). doi:10.1103/PhysRevLett.97.020801

39. Schneider, T., Peik, E., Tamm, Chr.: PRL 94, 230801 (2005)

40. Margolis, H.S., et al.: Science. 306, 1355 (2004). doi:10.1126/science.1105497

41. Rosenband, T., et al.: Science 319, 1808 (2008)

42. Linfield, R.P., Colavita, M.M., Lane, B.F.: ApJ. 554, 505 (2001). doi:10.1086/321372

43. Mendes, V.B., Pavlis, E.C.: Geoph. Res. Lett. 31, 14 (2004)

44. Mendes, V.B., et al.: Geophys. Res. Lett. 29, 1414 (2002). doi:10.1029/2001GL014394

45. Richardson, R., Chan, J.: Advanced Stirling radioisotope generator development. In: First Annual NASA Science Technology Conference (2007)

46. Sanders, H.M.: Updated Ariane 5 performances for escape, elliptical and circular missions. ESA/ESOC MAS Working Paper No. 360 (1994) 